\title{
A Tropical Stochastic Skeleton Model for the MJO, EI Niño, and Dynamic Walker Circulation: A Simplified GCM
}

\author{
SULIAN THUAL \\ Department of Mathematics, and Center for Atmosphere Ocean Science, Courant Institute of Mathematical Sciences, \\ New York University, New York, New York
}

\begin{abstract}
ANDREW J. MAJDA
Department of Mathematics, and Center for Atmosphere Ocean Science, Courant Institute of Mathematical Sciences, New York University, New York, New York, and Center for Prototype Climate Modeling, New York University Abu Dhabi, Saadiyat Island, Abu Dhabi, United Arab Emirates
\end{abstract}

\section{NAN CHEN}

Department of Mathematics, and Center for Atmosphere Ocean Science, Courant Institute of Mathematical Sciences, New York University, New York, New York

(Manuscript received 1 May 2018, in final form 27 August 2018)

\begin{abstract}
A simple dynamical stochastic model for the tropical ocean atmosphere is proposed that captures qualitatively major intraseasonal to interannual processes altogether including El Niño-Southern Oscillation (ENSO), the Madden-Julian oscillation (MJO), the associated wind bursts, and the background dynamic Walker circulation. Such a model serves as a prototype "skeleton" for general circulation models (GCMs) that solve similar dynamical interactions across several spatiotemporal scales but usually show common and systematic biases in representing tropical variability as a whole. The most salient features of ENSO, the wind bursts, and the MJO are captured altogether including their overall structure, evolution, and fundamental interactions in addition to their intermittency, diversity, and energy distribution across scales. Importantly, the intraseasonal wind bursts and the MJO are here solved dynamically, which provides their upscale contribution to the interannual flow as well as their modulation in return in a more explicit way. This includes a realistic onset of El Niño events with increased wind bursts and MJO activity starting in the Indian Ocean to the western Pacific and expanding eastward toward the central Pacific, as well as significant interannual modulation of the characteristics of intraseasonal variability. A hierarchy of cruder model versions is also analyzed in order to highlight fundamental concepts related to the treatment of multiple time scales, main convective nonlinearities, and the associated stochastic convective parameterizations. The model developed here also should be useful to diagnose, analyze, and help eliminate the strong tropical biases that exist in current operational models.
\end{abstract}

\section{Introduction}

El Niño-Southern Oscillation (ENSO) is a major climate signal on Earth with dramatic worldwide ecological and social impacts. It consists of alternating periods of anomalously warm El Niño and cold La Niña conditions every 2-7 years, with considerable irregularity in amplitude, duration, temporal evolution, and spatial structure of these events. Its dynamics in the equatorial Pacific result largely from coupled interactions between

Corresponding author: Nan Chen, nan.chen@nyu.edu the ocean and atmosphere at interannual time and planetary scales (Neelin et al. 1998; Clarke 2008). One salient yet not fully understood feature of ENSO is its interaction with atmospheric processes on a vast range of spatiotemporal scales. For instance, a broad range of intraseasonal atmospheric disturbances in the tropics may be considered as possible triggers to El Niño or La Niña events (Kleeman 2008). Those atmospheric disturbances are usually generally denoted as westerly wind bursts (WWBs) or easterly wind bursts (EWBs), though they may have different origins such as tropical cyclones or midlatitude cold surges as well as the 
convective envelope of the Madden-Julian oscillation (MJO), among others (Harrison and Vecchi 1997; Vecchi and Harrison 2000; Kiladis et al. 2009). In particular, westerly wind bursts reach strong intensity levels over the western Pacific warm pool during the onset of El Niño events (Tziperman and Yu 2007). The MJO is the dominant component of intraseasonal variability in the tropics and plays an important role in the generation of wind bursts (Madden and Julian 1971, 1994). In the troposphere, it begins as a standing wave in the Indian Ocean and propagates eastward as an equatorial planetaryscale wave across the western Pacific Ocean at a speed of around $5 \mathrm{~m} \mathrm{~s}^{-1}$ (Zhang 2005). The MJO features both westerly and easterly wind bursts at the same time within its convective envelope (Puy et al. 2016) and is also more prominent during the onset of El Niño events (Kleeman and Moore 1997; Moore and Kleeman 1999; Zhang and Gottschalck 2002; McPhaden et al. 2006; Hendon et al. 2007). In addition to the above features, ENSO dynamics are also closely linked to the destabilization of the background equilibrium circulation in the equatorial Pacific and the so-called Walker circulation that consists of an overturning zonal-vertical atmospheric circulation along with a zonal seesaw gradient of sea surface temperatures and thermocline depth in the ocean (Clarke 2008).

The interaction between ENSO, the wind bursts, and the Walker circulation is the focus of various observational initiatives and modeling studies. The challenges to deal with are twofold. First, general circulation models (GCMs) have common and systematic biases in representing ENSO, the intraseasonal atmospheric variability, and the background circulation in the tropics altogether (Lin et al. 2006; Kim et al. 2009; Wittenberg et al. 2006, 2014; Guilyardi et al. 2016). In these models, computing resources are significantly limited. For example, the spatial resolution is only up to $\approx 10-100 \mathrm{~km}$, and therefore several important small scales are unresolved or parameterized according to various recipes. In regards to tropical convection, unresolved processes at smaller scales such as deep convective clouds show some particular features in space and time, such as high irregularity, high intermittency, and low predictability. Recent improvements suggest that suitable stochastic parameterizations are good candidates to account for those processes while remaining computationally efficient (Majda et al. 2008; Palmer 2012; Weisheimer et al. 2014; Deng et al. 2015; Goswami et al. 2017a,b; Christensen et al. 2017). Second, there is a general lack of theoretical understanding of the dynamical interactions between ENSO and intraseasonal variability in GCMs. On the other hand, insight has been gained from simple and intermediate models for ENSO that have more tractable dynamics, are more computationally efficient, and allow for more detailed and systematic statistical analysis (e.g., Moore and Kleeman 1999; Neelin and Zeng 2000; Zeng et al. 2000; Jin et al. 2007; Gushchina and Dewitte 2011; Chen et al. 2015; Thual et al. 2016). For example, those models indicate the multiplicative noise features that can exist when wind bursts depend on the state of the equatorial Pacific system (Eisenman et al. 2005; Tziperman and Yu. 2007; Gebbie et al. 2007; Lopez et al. 2013). Yet, in those models, wind bursts are usually not resolved dynamically but are described by simple stochastic parameterizations that prescribe the wind burst amplitudes, durations, and/or propagation. As a result, those simple models do not resolve some of the important wind bursts details such as their dynamical evolution and origins.

In the present article, a simplified dynamical stochastic model is developed for the intraseasonal to interannual variability in the tropics and background circulation. The model is denoted hereafter tropical stochastic skeleton general circulation model (TSS-GCM). The present model serves as a prototype "skeleton" for GCMs that solve similar dynamical interactions across several spatiotemporal scales. As compared to conventional GCMs, the present TSS-GCM includes simple tractable dynamics with a minimal number of processes and parameters and is computationally very uncostly. Importantly, while conventional GCMs have common and systematic biases in representing tropical variability as a whole, the TSS-GCM succeeds in capturing major intraseasonal to interannual processes as well as their fundamental interactions in qualitative fashion. First, at intraseasonal time scales, the TSS-GCM captures dynamical wind bursts with realistic intermittency, localization, lifespan, convective features, energy distribution across scales, and generation from various sources including from the MJO. In particular, the main features of the MJO are recovered, including its eastward propagation, structure, and organization into intermittent wave trains with growth and demise. Second, at interannual time scales, the TSS-GCM captures the overall structure and period of ENSO, in addition to its intermittency and diversity with El Niño events of varying strength and intensity. The associated dynamic background Walker circulation is also captured qualitatively. Third, and most important, the TSS-GCM captures the most salient interactions between ENSO, wind bursts, and the MJO. This includes a realistic onset of El Niño events with increased wind bursts and MJO activity starting in the Indian to western Pacific Oceans and expanding eastward toward the central Pacific. In return, the characteristics of wind bursts and the MJO are significantly modulated interannually by the underlying 
variations of sea surface temperatures associated with ENSO, as in nature. The TSS-GCM formulation provides such an upscale contribution of the wind bursts to the interannual flow and their modulation in return in an explicit and dynamical way.

The TSS-GCM introduced in the present article builds on a range of previous work by the authors. First, for the intraseasonal variability in the atmosphere Majda and Stechmann $(2009,2011)$ introduced a minimal dynamical model, the skeleton model, that captures for the first time the main features of the MJO. This includes the MJO eastward phase speed of $5 \mathrm{~m} \mathrm{~s}^{-1}$, peculiar dispersion relation with $d \omega / d k \approx 0$, and horizontal quadrupole structure, among others. The model depicts the MJO as a neutrally stable atmospheric wave that involves a simple interaction between planetary-scale dry dynamics and lower-tropospheric moisture as well as the planetary envelope of synoptic-scale convection/wave activity. In subsequent work, such an MJO skeleton model refined with a suitable stochastic convective parameterization has been shown to capture the intermittent generation of MJO events and their organization into waves trains with growth and demise (i.e., series of consecutive events) as in nature (Thual et al. 2014; Stachnik et al. 2015; Majda et al. 2018). The MJO skeleton model appears to be an excellent candidate for capturing dynamically the variability of intraseasonal wind bursts in simple fashion. In the present TSS-GCM, such a skeleton atmosphere with simple self-consistent nonlinear noise (Chen and Majda 2016a) is used with a simple multiple-time approach (Majda and Klein 2003) that allows us to derive approximate dynamics on both the intraseasonal and interannual time scales. Second, for the interannual variability in general, a simple ocean-atmosphere coupled model was developed recently that emphasizes the role of state-dependent wind bursts and realistically captures ENSO diversity, including the eastern Pacific moderate and occasional super El Niño (Thual et al. 2016). In this coupled model, stochastic wind bursts are coupled to otherwise deterministic, linear, and stable ocean-atmosphere dynamics: In fact, the wind bursts play the role of maintaining ENSO, which is fundamentally different from the Cane-Zebiak model (Zebiak and Cane 1987) and other nonlinear models that rely instead on internal instability. However, such a coupled model does not solve wind bursts dynamically, which is a usual feature of simple ENSO models as discussed above. Instead, it uses a simple stochastic parameterization to generate randomly both WWBs and EWBs from an identical white noise source. In the TSS-GCM from the present article, the coupled ocean-atmosphere dynamics from Thual et al. (2016) are included with, however, a more realistic depiction of wind bursts and their dynamical features directly from the coupled atmosphere skeleton model. Finally, note that the coupled ocean-atmosphere dynamics from Thual et al. (2016) have been largely extended in subsequent work in order to facilitate additional realistic features such as the occurrence of central Pacific El Niño events (Chen and Majda 2016b, $2017,2018)$ or the delayed super El Niño as of 2015/16 (Thual et al. 2018) in addition to the synchronization of ENSO to the seasonal cycle (Thual et al. 2017), among others. Although such processes are certainly important for ENSO, they will be omitted for the scope of the present article in order to document the inclusion of intraseasonal dynamics in the TSS-GCM in simpler fashion.

The present article is organized as follows. In section 2, we present the TSS-GCM used in this study, along with a hierarchy of cruder versions of the model used to introduce progressively fundamental concepts related to the treatment of multiple time scales, main convective nonlinearities, and associated stochastic parameterizations. In section 3, we analyze the main properties of the TSS-GCM and its versions, including their depiction of the intraseasonal wind bursts and $\mathrm{MJO}$ variability and interannual ENSO variability as well as the dynamic Walker circulation. Section 4 is a discussion with concluding remarks.

\section{Formulation of the TSS-GCM}

In this section, we formulate the TSS-GCM used in the present study. To formulate the model, first, a starting deterministic atmosphere and ocean are considered (Majda and Stechmann 2009; Thual et al. 2016; Chen and Majda 2016b, 2017, 2018). In particular, the deterministic atmosphere is decomposed into an intraseasonal and interannual flow following a simple multipletime approach (Majda and Klein 2003). Next, simplified versions of the TSS-GCM are derived: a crude interannual model and crude intraseasonal model. Such cruder model versions differ from the complete TSS-GCM by their simplified representations of intraseasonal processes and are introduced first for dynamical insight. Finally, the complete TSS-GCM is formulated as well as a more complete version with a dynamic Walker circulation. At the end of the section, an overview and intercomparison of the features of each model version is provided, as well as their contrast with conventional GCMs.

\section{a. Starting deterministic atmosphere}

To derive the TSS-GCM, we consider first the starting deterministic skeleton model atmosphere from Majda and Stechmann (2009). Such a skeleton model captures 
the main features of intraseasonal variability in general in the tropics, including importantly the MJO eastward propagation, peculiar dispersion relation, and quadrupole structure, among others (Majda and Stechmann 2009, 2011). Such a model reads as follows:

$$
\begin{aligned}
\partial_{t} u-y v-\partial_{x} \theta & =0, \\
y u-\partial_{y} \theta & =0, \\
\partial_{t} \theta-\left(\partial_{x} u+\partial_{y} v\right) & =\bar{H} a-s^{\theta}, \\
\partial_{t} q+\bar{Q}\left(\partial_{x} u+\partial_{y} v\right) & =-\bar{H} a+s^{q}+E_{q}, \\
\partial_{t} a & =\Gamma q a .
\end{aligned}
$$

In the above model, $x$ is zonal direction, $y$ is meridional direction, and $t$ is intraseasonal time. The $u, v$ are zonal and meridional winds, $\theta$ is potential temperature, $q$ is lower-level moisture, and $a$ is the planetary envelope of convective activity. All variables are anomalies except $a>0$. The $a$ in particular is a collective (i.e., integrated) representation of the unresolved convection/wave activity details occurring at synoptic scale, always acting as a planetary source of heating and drying (hence $a>0$ ). A key idea in the above model is that environmental moisture $q$ influences the growth/decay of convective activity in general as well as their planetary envelope $a$. Note that as compared to Majda and Stechmann (2009), we have added in Eq. (1) the contribution of latent heat release $E_{q}$ in order to allow coupling with the ocean. The $s^{\theta}$ and $s^{q}$ are constant external sources of cooling and moistening, respectively, and $\bar{Q}$ and $\Gamma$ are parameters.

Next, the above system is decomposed into an intraseasonal atmosphere and interannual background mean atmosphere. A general motivation for this is to derive approximate solutions for the slowly varying fluctuations relevant to ENSO. For this, we assume that such slowly varying fluctuations exist on the interannual time, in addition to the rapidly varying fluctuations on the intraseasonal time scale (Majda and Klein 2003). Details on the derivation are provided in appendix A. The flow in Eq. (1) is decomposed as $a=\bar{a}+a^{\prime}$ in standard notations from turbulence theory and similarly for $u, v, \theta$, and $q$. First, the resulting intraseasonal deterministic atmosphere reads as follows:

$$
\begin{aligned}
\partial_{t} u^{\prime}-y v^{\prime}-\partial_{x} \theta^{\prime} & =0, \\
y u^{\prime}-\partial_{y} \theta^{\prime} & =0, \\
\partial_{t} \theta^{\prime}-\left(\partial_{x} u^{\prime}+\partial_{y} v^{\prime}\right) & =\bar{H} a^{\prime}, \\
\partial_{t} q^{\prime}+\bar{Q}\left(\partial_{x} u^{\prime}+\partial_{y} v^{\prime}\right) & =-\bar{H} a^{\prime}, \\
\partial_{t} a^{\prime} & =\Gamma q^{\prime}\left(\bar{a}+a^{\prime}\right),
\end{aligned}
$$

which models intraseasonal fluctuations in general such as the MJO as well as other planetary convectively coupled waves. Such a system is dynamically similar to the starting skeleton model from Majda and Stechmann (2009, 2011), though the background $\bar{a}$ (with $\bar{a} \geq 0$ ) here varies interannually as modulated by the ocean conditions (see hereafter). Note that the intraseasonal contribution of latent heat release $E_{q}^{\prime}$ is small and omitted here (see appendix A). Next, the interannual deterministic atmosphere reads as follows:

$$
\begin{aligned}
-y \bar{v}-\partial_{x} \bar{\theta} & =0, \\
y \bar{u}-\partial_{y} \bar{\theta} & =0, \\
-\left(\partial_{x} \bar{u}+\partial_{y} \bar{v}\right) & =\bar{H} \bar{a}-s^{\theta}, \\
-\bar{Q}\left(\partial_{x} \bar{u}+\partial_{y} \bar{v}\right) & =\bar{H} \bar{a}+s^{q}+E_{q}, \\
\bar{H} \bar{a} & =\left(E_{q}+s^{q}-\bar{Q} s^{\theta}\right) /(1-\bar{Q}),
\end{aligned}
$$

which depicts the interannual adjustment of the atmosphere to the ocean conditions. In particular, there are no time derivatives in the system from Eq. (3) that are assumed to remain in balance with the underlying ocean on the slow interannual time scale where the forcing $\bar{E}_{q}$ (noted here $E_{q}$ for brevity) varies (Gill 1980). Such an interannual atmosphere is identical to the one from Thual et al. (2016), though it is derived here from a different method (multiple time scales instead of single time scale approach; see appendix A). Note that wind divergence in Eq. (3) can alternatively be expressed as

$$
-\left(\partial_{x} \bar{u}+\partial_{y} \bar{v}\right)=\left(E_{q}+s^{q}-s^{\theta}\right) /(1-\bar{Q}) .
$$

For instance, unbalanced sources of heating/moistening $E_{q}+s^{q}-s^{\theta} \neq 0$ force a background interannual circulation similar to the Walker circulation in nature (Chen and Majda 2016b; Ogrosky and Stechmann 2015), as discussed hereafter.

\section{b. Starting ocean, SST, and couplings}

Next, the above deterministic atmosphere [Eqs. (2) and (3)] is coupled to the ocean. For this, we consider a simple shallow-water ocean and sea surface temperature (SST) budget that retains a few essential processes relevant to ENSO interannual variability. Because the ocean dynamics are essentially interannual, no multipletime approach is considered here. The starting ocean [Eq. (5)], SST budget [Eq. (6)], and couplings [Eq. (7)] are identical to the ones of Thual et al. (2016). They read as follows:

$$
\begin{aligned}
\partial_{t} U-\epsilon c_{1} Y V+\epsilon c_{1} \partial_{x} H & =\epsilon c_{1} \tau_{x}, \\
Y U+\partial_{Y} H & =0, \\
\partial_{t} H+\epsilon c_{1}\left(\partial_{x} U+\partial_{Y} V\right) & =0, \\
\partial_{t} T=-\epsilon c_{1} \zeta E_{q}+\epsilon c_{1} \eta H, & \text { and }
\end{aligned}
$$


(a) Complete TSS-GCM Model

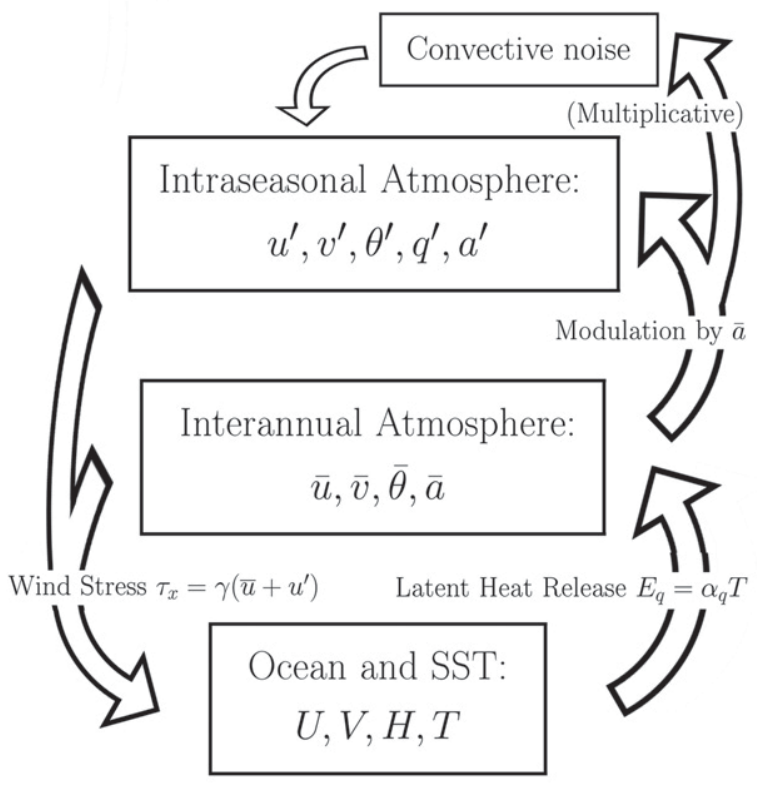

(b) Crude Interannual Model

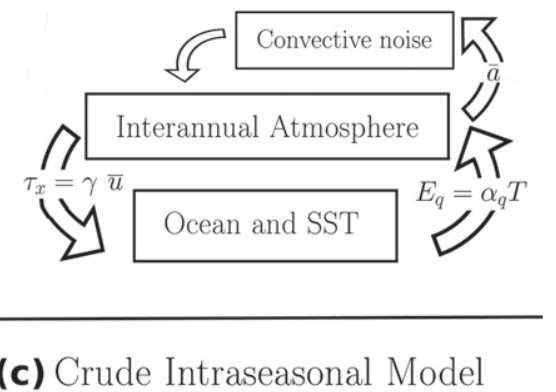

(c) Crude Intraseasonal Model

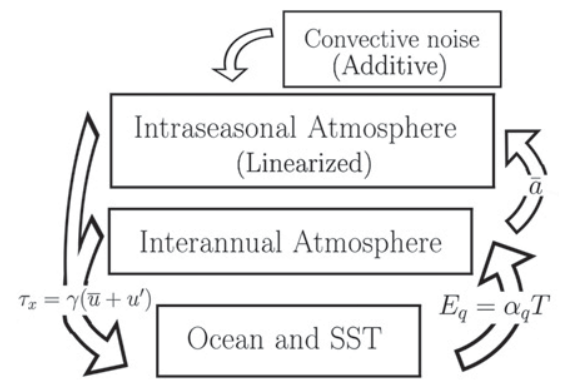

FIG. 1. (a) Sketch of the couplings between the intraseasonal atmosphere, interannual atmosphere, ocean, and SST as well as convective noise in the TSS-GCM. Sketch of the couplings in the (b) crude interannual and (c) crude intraseasonal model versions.

$$
\begin{aligned}
\tau_{x} & =\gamma\left(\bar{u}+u^{\prime}\right), \\
E_{q} & =\alpha_{q} T .
\end{aligned}
$$

In the above Eqs. (5)-(7), $Y$ is meridional direction in the ocean, $U$ and $V$ are zonal and meridional currents, $H$ is thermocline depth, $\tau_{x}$ is zonal wind stress, and $T$ is SST. Only a few processes deemed most important are retained in the SST budget from Eq. (6), such as dissipation by latent heat losses and the so-called thermocline feedback (An and Jin 2001; Thual et al. 2016). Note that the ocean covers the equatorial Pacific domain only with boundary conditions at the western and eastern edges (see hereafter). The above system includes a minimal number of parameters: Froude number $\epsilon, c_{1}, \zeta$, $\eta, \gamma$, and $\alpha_{q}$ (see details in appendix B).

A few important remarks can be made on the coupling between the above ocean and SST model from Eqs. (5)-(7) and the intraseasonal and interannual atmospheres from Eqs. (2) and (3). Figure 1a shows a sketch of the couplings in the complete TSS-GCM. First, the ocean, SST, and interannual atmosphere [Eqs. (5)-(7), (3)] are coupled through latent heat release $E_{q}=\alpha_{q} T$ that forces an atmosphere circulation. The resulting zonal wind stress $\tau_{x}$ in return forces an ocean circulation that modifies the sea surface temperatures through thermocline depth anomalies $H$. In the absence of the intraseasonal atmosphere, such a coupled interannual oceanatmosphere system is linear, deterministic, and stable and simulates a dissipated ENSO cycle with realistic period of $\approx 4.5 \mathrm{yr}$ and overall structure (see supplemental information of Thual et al. 2016). Second, the intraseasonal atmosphere [Eq. (2)] is the starting skeleton model from Majda and Stechmann (2009) and intends to model the main features of the MJO. Here, such an intraseasonal atmosphere is fully coupled to the interannual atmosphere-ocean system. The intraseasonal wind bursts $u^{\prime}$ force the ocean through the wind stress $\tau_{x}$ in Eq. (7), and the ocean conditions modulate the intraseasonal atmosphere through interannual convective activity $\bar{a}$ in Eq. (2). Notably, the intraseasonal atmosphere plays the role of triggering ENSO in the otherwise-dissipated ocean-atmosphere system, which is fundamentally different from the Cane-Zebiak (Zebiak and Cane 1987) model and other nonlinear models that rely instead on internal ocean instability. Finally, as shown in Fig. 1a, in the complete TSS-GCM, convective noise is added to the intraseasonal atmosphere that depends on the interannual convective activity $\bar{a}$ (i.e., is multiplicative). The details of this convective stochastic parameterization will be introduced hereafter.

\section{c. Crude interannual atmosphere}

In the next subsections, in order to derive the complete TSS-GCM, we will first consider a hierarchy of cruder model versions. Those crude model versions have simplified dynamics and/or stochastics that allow us to 
understand the underlying processes in the more realistic complete TSS-GCM. We introduce here first a crude interannual model, followed by a crude intraseasonal model, before presenting the complete TSS-GCM.

Figure $1 b$ shows a sketch of the couplings in the crude interannual model. In the crude interannual model, the intraseasonal dynamics are omitted in favor of a simple stochastic parameterization of intraseasonal wind bursts. This follows the prototype of many simple or intermediate models depicting the relationship between ENSO and wind bursts, where intraseasonal dynamics are not solved explicitly (e.g., Moore and Kleeman 1999; Eisenman et al. 2005; Jin et al. 2007; Chen et al. 2015; Thual et al. 2016). The crude interannual model reads as follows:

$$
\begin{aligned}
-y \bar{v}-\partial_{x} \bar{\theta} & =0, \\
y \bar{u}-\partial_{y} \bar{\theta} & =0, \\
-\left(\partial_{x} \bar{u}+\partial_{y} \bar{v}\right) & =\bar{H} \bar{a}-s^{\theta}, \\
-\bar{Q}\left(\partial_{x} \bar{u}+\partial_{y} \bar{v}\right) & =\bar{H} \bar{a}+s^{q}+E_{q},
\end{aligned}
$$

along with

$$
\begin{aligned}
\partial_{t} \bar{a} & =-\lambda(\bar{a}-\hat{a})+\sqrt{\lambda \hat{a} \bar{a}} \dot{W}, \\
\bar{H} \hat{a} & =\left(E_{q}+s^{q}-\bar{Q} s^{\theta}\right) /(1-\bar{Q}),
\end{aligned}
$$

and with no intraseasonal fluctuations; that is, $u^{\prime}, v^{\prime}, \theta^{\prime}, q^{\prime}, a^{\prime}=0$. Meanwhile, the ocean and SST are identical to the ones in the previous sections [Eqs. (5)-(7)]. Equation (9) is a simple stochastic differential equation (SDE) for intraseasonal variability (Chen and Majda 2016a) where the background convective activity $\bar{a}$ is perturbed by a white noise source $\dot{W}$ and relaxes to $\hat{a}$ at a rate $\lambda=(30 \text { day })^{-1}$. Note that without noise perturbations and after relaxation (i.e., for $\bar{a}=\hat{a}$ ) we would retrieve the starting deterministic interannual atmosphere from Eq. (3). Importantly, the SDE involves a multiplicative noise that ensures that $\bar{a} \geq 0$ (as long as $\hat{a} \geq 0$ ) in the model, consistent with the definition of convective activity in previous sections. In fact, the equilibrium probability distribution of $\bar{a}$ relaxes to a gamma distribution:

$$
P(\bar{a})=\frac{1}{\mu^{k} G(k)} \bar{a}^{k-1} \exp (-\bar{a} / \mu),
$$

with mean $\hat{a}$ and for which $\bar{a} \geq 0$ as shown in Fig. $2 \mathrm{~d}$, with here parameters $k=2$ and $\mu=\hat{a} / 2$.

\section{d. Crude intraseasonal atmosphere}

We now formulate the crude intraseasonal model, with a sketch of couplings shown in Fig. 1c. As compared to the crude interannual model presented above, the crude intraseasonal model captures the dynamical details of intraseasonal variability. Such details are, however, simplified to some extent because some fundamental convective nonlinearities and associated noise features are missing that will be introduced hereafter in the complete TSS-GCM. The crude intraseasonal model reads as follows:

$$
\begin{aligned}
\left(\partial_{t}+d_{u}\right) u^{\prime}-y v^{\prime}-\partial_{x} \theta^{\prime} & =0, \\
y u^{\prime}-\partial_{y} \theta^{\prime} & =0, \\
\left(\partial_{t}+d_{u}\right) \theta^{\prime}-\left(\partial_{x} u^{\prime}+\partial_{y} v^{\prime}\right) & =\bar{H} a^{\prime}, \\
\left(\partial_{t}+d_{q}\right) q^{\prime}+\bar{Q}\left(\partial_{x} u^{\prime}+\partial_{y} v^{\prime}\right) & =-\bar{H} a^{\prime}+\sigma_{q} \dot{W}_{q}, \\
\left(\partial_{t}+d_{a}\right) a^{\prime} & =\Gamma q^{\prime} \bar{a},
\end{aligned}
$$

in addition to the interannual atmosphere, ocean, and SST that are identical to the ones in previous sections [Eqs. (3), (5)-(7)]. As compared to the starting deterministic intraseasonal atmosphere from Eq. (2), moisture is perturbed in Eq. (11) by an additive white noise source $\dot{W}_{q}$, and uniform dissipations $d_{u}, d_{q}, d_{a}$ are added consistent with the noise-dissipation energy balance (Hottovy and Stechmann 2015; Stechmann and Hottovy 2017). Here, $d_{u}, d_{q}, d_{a}=(30 \text { day })^{-1}$, which is a natural dissipation time scale for intraseasonal variability. In addition, for simplicity, the evolution of convective activity $a^{\prime}$ from Eq. (2) is here linearized around the interannual mean value $\bar{a}$ (and remains approximately linear for $\bar{a}$ varying on the slower interannual time scale). As a result, despite involving simpler linearized dynamics, an important caveat of the present crude intraseasonal model is that total convective activity $\bar{a}+a^{\prime}$ is not always positive (though $\bar{a}$ remains positive), which is a deficiency compared with the starting deterministic skeleton model formulation from Eq. (1) (Majda and Stechmann 2009, 2011).

\section{e. Complete TSS-GCM}

We now formulate the complete TSS-GCM, with a sketch of couplings shown in Fig. 1a. The TSS-GCM includes all the features from the starting deterministic ocean and atmosphere, with in addition important design elements already introduced above with the crude interannual and crude intraseasonal models (Figs. 1b,c). As compared to those crude models, the complete TSSGCM retains some fundamental nonlinearities and multiplicative noise features associated with convection in nature, which are common to conventional GCMs. As shown hereafter, such a convective parameterization allows the complete TSS-GCM to capture more realistically some important features of wind bursts in 


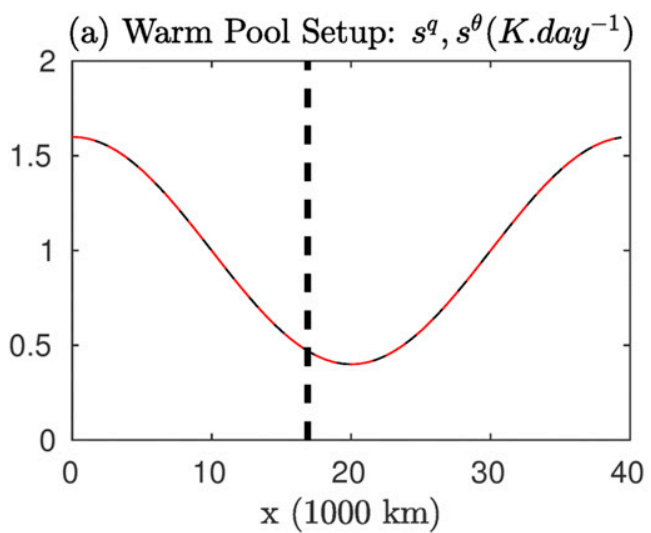

(c) Thermocline Feedback: $\eta(x)$

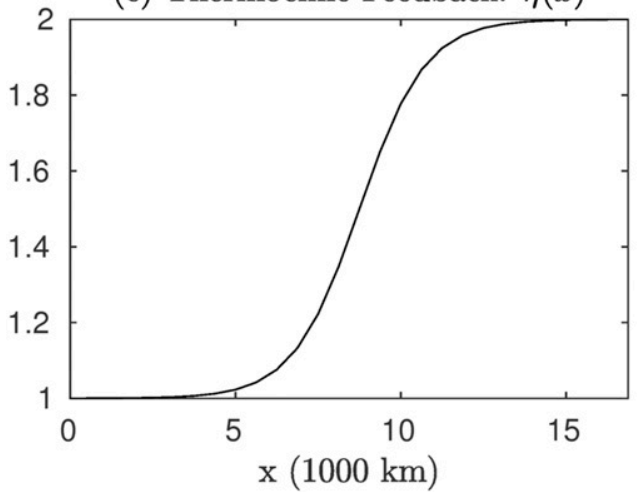

(b) Walker Circulation Setup: $s^{q}, s^{\theta}\left(K . d a y^{-1}\right)$

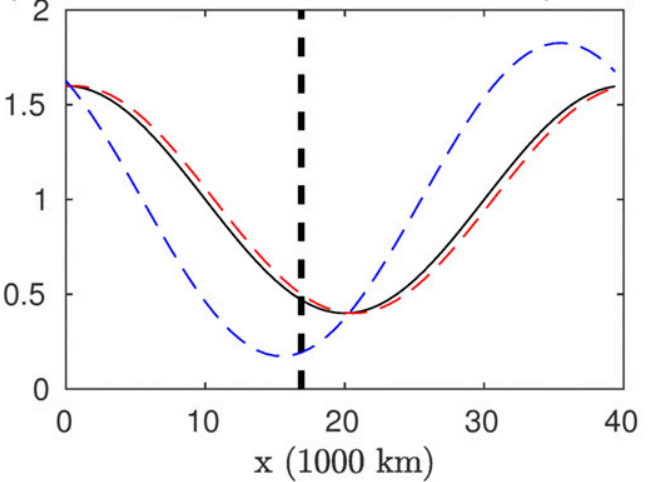

(d) Gamma Distribution

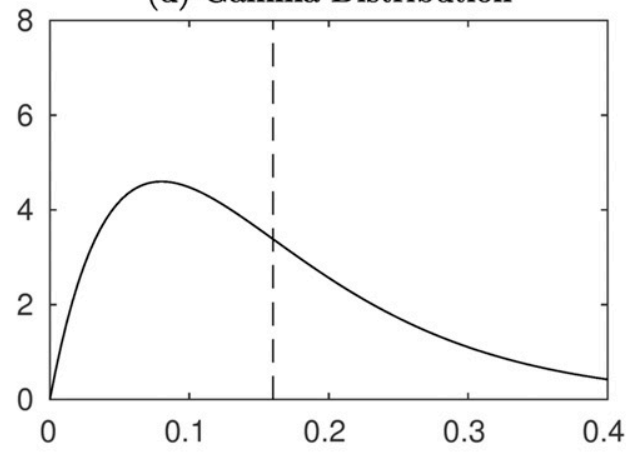

FIG. 2. Zonal profiles of external moisture source $s^{q}$ (black; $\mathrm{K} \mathrm{day}^{-1}$ ) and cooling source $s^{\theta}\left(\mathrm{red} ; \mathrm{K} \mathrm{day}^{-1}\right.$ ) at the equator for (a) the TSS-GCM and (b) the TSS-GCM with dynamic Walker circulation [in addition to $s^{m}=\left(s^{q}-\bar{Q} s^{\theta}\right) /(1-\bar{Q})$ in blue], around the equatorial belt as a function of zonal position $x(1000 \mathrm{~km})$. (c) Zonal profile of the thermocline feedback parameter $\eta(x)$ in the equatorial Pacific (nondimensional). (d) Equilibrium gamma probability distribution for convective activity (nondimensional).

nature such as their sharpness and intensity in addition to conserving a positive convective activity. The complete TSS-GCM instraseasonal atmosphere reads as follows:

$$
\begin{aligned}
&\left(\partial_{t}+d_{u}\right) u^{\prime}-y v^{\prime}-\partial_{x} \theta^{\prime}=0, \\
& y u^{\prime}-\partial_{y} \theta^{\prime}=0, \\
&\left(\partial_{t}+d_{u}\right) \theta^{\prime}-\left(\partial_{x} u^{\prime}+\partial_{y} v^{\prime}\right)=\bar{H} a^{\prime}, \\
&\left(\partial_{t}+d_{q}\right) q^{\prime}+\bar{Q}\left(\partial_{x} u^{\prime}+\partial_{y} v^{\prime}\right)=-\bar{H} a^{\prime}+\sigma_{q} \dot{W}_{q}, \\
& \partial_{t} a^{\prime}=\Gamma q^{\prime}\left(\bar{a}+a^{\prime}\right)-\lambda a^{\prime}+\sqrt{\lambda\left(\bar{a}+a^{\prime}\right) \bar{a}} \dot{W}_{a},
\end{aligned}
$$

in addition to the interannual atmosphere, ocean, and SST that are identical to the ones in previous sections [Eqs. (3), (5)-(7)]. As compared to the starting deterministic intraseasonal atmosphere from Eq. (2), we have added in Eq. (2) white noise sources terms $\dot{W}_{q}, \dot{W}_{a}$, and associated dissipations as in the crude intraseasonal atmosphere from Eq. (11), in addition to a suitable SDE for convective activity $a^{\prime}$ as in the crude intraseasonal atmosphere from Eq. (9). Such an SDE involves multiplicative noise ensuring that $a^{\prime}+\bar{a}>0$ in agreement with the starting deterministic skeleton model formulation from Eq. (1) (Majda and Stechmann 2009, 2011). In fact, the time tendency $\partial_{t} a^{\prime}$ in Eq. (12) is driven by $\Gamma q^{\prime}\left(\bar{a}+a^{\prime}\right)$ as well as $-\lambda a^{\prime}+\sqrt{\lambda\left(\bar{a}+a^{\prime}\right) \bar{a}} \dot{W}_{a}$, which both ensure that $a^{\prime}+\bar{a}>0$ when considered independently (Majda and Stechmann 2009, 2011; Chen and Majda 2016a), therefore $a^{\prime}+\bar{a}>0$ is ensured by splitting method. In addition, for $q^{\prime}=0$, the $a^{\prime}+\bar{a}$ relaxes to a gamma distribution as in Fig. 2 d.

\section{f. Complete TSS-GCM with dynamic Walker circulation}

A dynamic Walker circulation can be obtained for unbalanced external sources of cooling/moistening in any version of the TSS-GCM presented above (crude interannual, crude intraseasonal, or complete TSSGCM). Recall that wind divergence in Eq. (3) can alternatively be expressed as follows:

$$
-\left(\partial_{x} \bar{u}+\partial_{y} \bar{v}\right)=\left(E_{q}+s^{q}-s^{\theta}\right) /(1-\bar{Q}) .
$$


In Eq. (13), $E_{q}+s^{q}-s^{\theta} \neq 0$ forces a background interannual atmosphere circulation, which can arise either from latent heat release fluctuations $E_{q} \neq 0$ driven by the ocean Eqs. (5)-(7) or unbalanced external sources of cooling/moistening $s^{\theta} \neq s^{q}$. As shown hereafter, such a background circulation is representative of the dynamic Walker circulation in the equatorial Pacific marked by mean westward trade winds and an overturning circulation in the upper troposphere (Chen and Majda 2016b; Ogrosky and Stechmann 2015) as well as an equilibrium zonal gradient of SST and thermocline depth in the ocean.

In the TSS-GCM as well as the crude interannual and intraseasonal models introduced above, there is no dynamic Walker circulation, and the external sources of cooling/moistening $s^{\theta}$ and $s^{q}$ are representative of a simple background warm pool of cooling/moistening as shown in Fig. 2a. For simplicity, the external sources are balanced, that is, $s^{q}=s^{\theta}$ and are maximal at the western edge of the equatorial Pacific $(x=0)$ and minimal around the eastern edge $(x \approx 18000 \mathrm{~km})$ [see, e.g., Majda and Stechmann (2011); Thual et al. (2014) for a similar parameterization]. This accounts qualitatively for the increased convective activity over the Indian Ocean/western Pacific and decreased convective activity in the eastern Pacific, although the profiles are unrealistic over the Atlantic Ocean, where another increase in convective activity is not accounted for (Ogrosky and Stechmann 2015). In the TSS-GCM with dynamic Walker circulation, the external sources are instead unbalanced as in nature (Ogrosky and Stechmann 2015), that is, $s^{q} \neq s^{\theta}$ as shown in Fig. 2b. Such an unbalance forces the background circulation in Eq. (13). For this, we have slightly shifted the profile of $s^{\theta}$. Despite the apparent similarity between $s^{q}$ and $s^{\theta}$ in Fig. 2b, note that the quantity $s^{m}=\left(s^{q}-\bar{Q} s^{\theta}\right) /(1-\bar{Q})$ that appears in the expression of $\bar{H} \bar{a}$ in Eq. (3) shows large zonal variations and remains positive to ensure $\bar{a} \geq 0$. Finally, note that although $s^{\theta}$ and $s^{q}$ are here constant with time, their variations with seasons could be accounted for in a more complex setup (e.g., Thual et al. 2015).

\section{g. Intercomparison of model versions}

Here, we provide a summary and intercomparison of all model versions of the TSS-GCM. The main features of all model versions are listed in Table 1 and are also contrasted with the ones of conventional GCMs. Those features will be detailed hereafter in the next sections.

The features summarized in Table 1 are as follows: First, conventional GCMs that retain the full complexity of the ocean-atmosphere system typically show common and systematic biases in representing ENSO, MJO, and background circulation altogether (Lin et al. 2006;

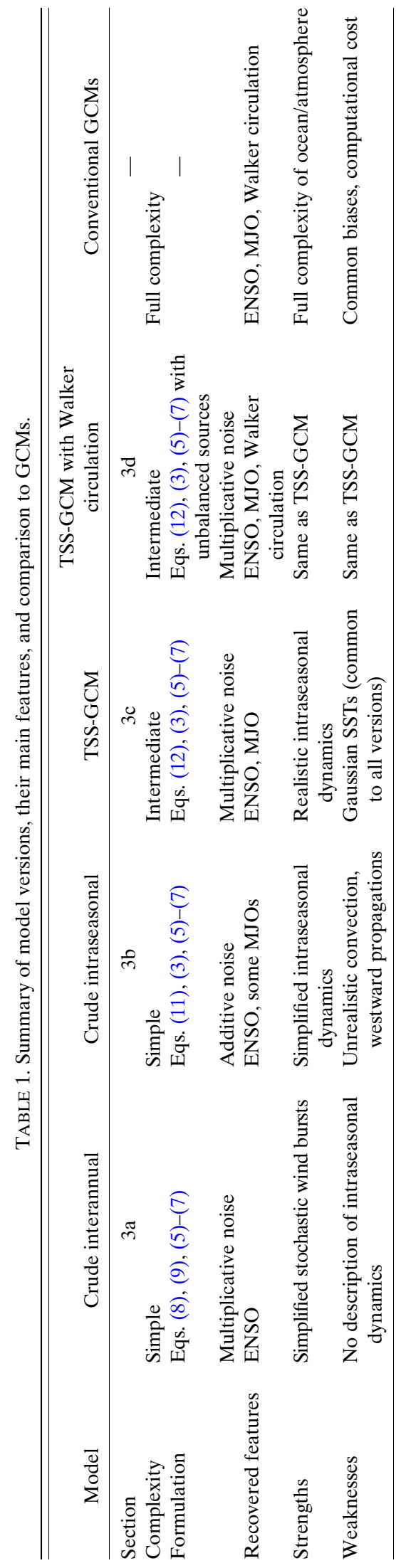


Kim et al. 2009; Wittenberg et al. 2006, 2014; Guilyardi et al. 2016). This may include biases for the background mean state, ENSO intermittency, and diversity as well as its non-Gaussian statistics, in addition to biases for the MJO amplitude, duration, and propagation (with often a weak or even absent MJO).

Second, the complete TSS-GCM (section 3b; Fig. 1a) in comparison shows great skill at capturing qualitatively the above processes and is computationally much less costly. Recovered features include an irregular and intermittent ENSO cycle with El Niño events of varying strength and intensity, in addition to intermittent MJO events and wind bursts that are realistically confined to the western Pacific/Indian Ocean region of convection and expand to the central Pacific during the onset of El Niño events. The model, however, reproduces unrealistic Gaussian SST statistics, which is also a common deficiency of all model versions as well as GCMs. In addition to this, the careful choice of SDE with multiplicative noise ensures that convective activity remains positive (Chen and Majda 2016a), consistent with the original formulation of such a variable in earlier work (Majda and Stechmann 2009, 2011). As compared to those earlier works, note that only a few additional parameters (dissipations and noise amplitude) need to be specified for the SDE.

Third, the TSS-GCM with dynamic Walker circulation (section 3d) is obtained from the complete TSSGCM simply by imposing unbalanced external sources of cooling/moistening, that is, $s^{\theta} \neq s^{q}$, which results in a cold tongue/warm pool region with associated cooling/ heating in the ocean and convection/subsidence in the atmosphere. Note that such a dynamic Walker circulation can also be obtained in the crude interannual or crude intraseasonal models by imposing $s^{\theta} \neq s^{q}$.

Next, in the crude intraseasonal model (section 3b; Fig. 1b) the atmosphere is simplified in terms of noise source and main nonlinearities. Such a crude model captures both ENSO and MJO in simple fashion but misses important convective details such as sharp and intense wind bursts and does not ensure a positive convective activity. In addition, the simulated intraseasonal variability is dominated by excessive power from moist westward-propagating Rossby waves and a weaker MJO in comparison. Finally, in the crude interannual model (section 3a; Fig. 1c) there are no intraseasonal atmospheric fluctuations but instead simple stochastic perturbations of the background convective activity $\bar{a}$, which is a prototype for most simple ENSO models with stochastic wind bursts (Moore and Kleeman 1999; Eisenman et al. 2005; Jin et al. 2007; Chen et al. 2015; Thual et al. 2016). This allows the model to generate ENSO variability in simple fashion, although there is no intraseasonal dynamics. In addition to this, note that the earlier ENSO model from Thual et al. (2016) and its subsequent improvements (Chen and Majda 2016b, 2017, 2018; Thual et al. 2017) all follow the same prototype as the crude interannual model with no intraseasonal dynamics. Those model versions have important features that are usually missing in GCMs such as realistic non-Gaussian SST statistics and central Pacific El Niño events as well as an ENSO seasonal synchronization, among others. Such features are certainly important for ENSO but are omitted for the scope of the present article.

In the next section, we analyze in detail the main features of the TSS-GCM as well as its versions as summarized in Table 1. Appendix B provides additional technical details on the model formulation and numerical solving algorithm.

\section{El Niño, the MJO, and the dynamic Walker circulation in the TSS-GCM}

In this section, we show results from numerical experiments with the TSS-GCM presented in previous section. For clarity and consistency with the previous section, we introduce here the main features of each model version in order of increasing complexity: crude interannual, crude intraseasonal, complete TSS-GCM, and complete TSSGCM with dynamic Walker circulation.

\section{a. Crude interannual model}

We show here solutions of the crude interannual model [see Fig. 1b and Eqs. (5)-(9) for its formulation]. In the crude interannual model, the intraseasonal atmosphere dynamics are omitted in favor of a simple stochastic parameterization with multiplicative features. This follows the prototype of many simple or intermediate models that describe the relationship between ENSO and wind bursts, in which intraseasonal dynamics are not solved explicitly (e.g., Moore and Kleeman 1999; Eisenman et al. 2005; Jin et al. 2007; Thual et al. 2016).

Figure 3 shows solutions of the crude interannual model. This includes the time series of $T_{E}$ the average of SST anomalies in the eastern half of the equatorial $\mathrm{Pa}$ cific (Fig. 3a), as well as the time series of convective activity $\bar{H} \bar{a}$ at the western edge of the Pacific (Fig. 3b). The $T_{E}$ is a good indicator of El Niño variability in the model because of its possible comparison to, for example, the observed Niño-3 SST index. The model simulates an ENSO cycle that is sustained, irregular, and intermittent, as in nature (Clarke 2008). While the evolution of $T_{E}$ is essentially interannual, the evolution of $\bar{H} \bar{a}$ is both intraseasonal and interannual (cf. 1-yr 
(a) $T_{E}$

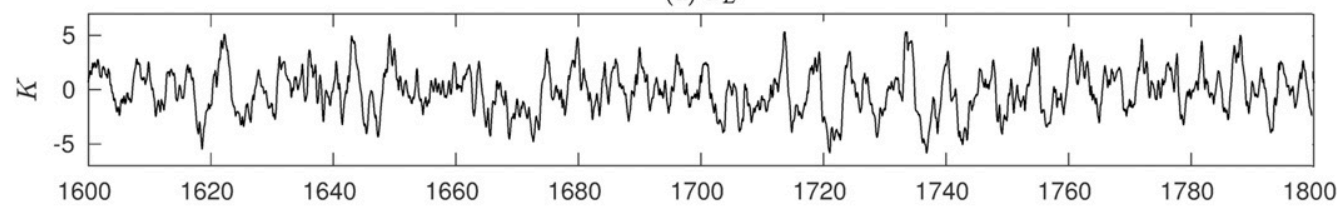

(b) $\bar{H} \bar{a}$
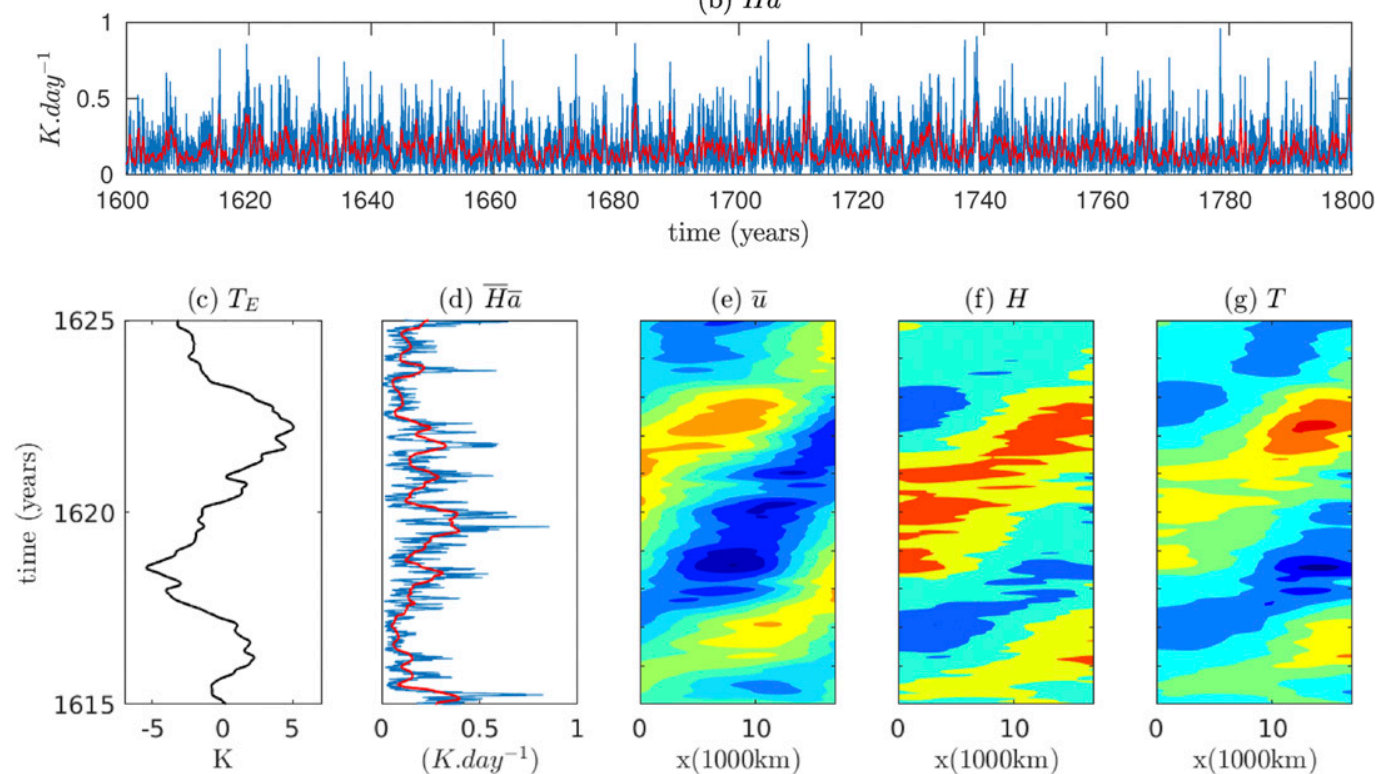

(d) $\bar{H} \bar{a}$

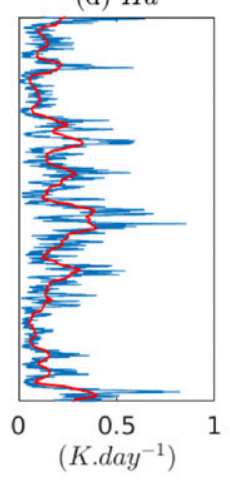

(e) $\bar{u}$

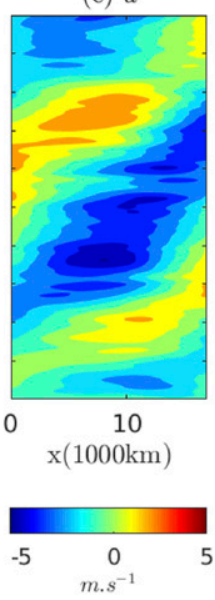

(f) $H$

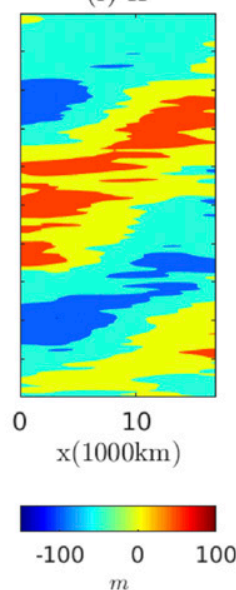

(g) $T$

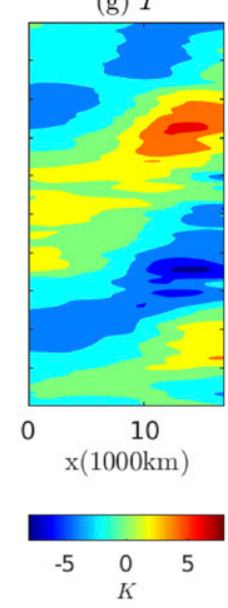

FIG. 3. Solutions of the crude interannual model. Time series of (a) $T_{E}$ the average of SSTs at the equator in the eastern half of Pacific (K) and (b) interannual convective activity $\bar{H} \bar{a}\left(\mathrm{~K} \mathrm{day}^{-1}\right)$ at the equator and at the western edge of the Pacific $(x=0)$. The red line in (b) is a 1-yr moving average. (c),(d) Repeats of the time series over a shorter period. Hovmöller diagrams of interannual (e) zonal winds $\bar{u}\left(\mathrm{~m} \mathrm{~s}^{-1}\right)$, (f) thermocline depth $H(\mathrm{~m})$, and (g) SST $T(\mathrm{~K})$ at the equator, as a function of zonal position and time (years).

moving average; red) consistent with the SDE parameterization in Eq. (9). This illustrates the simple mechanisms for the generation of interannual variability in the model that results from the integration of noise: The interannual ocean-atmosphere system is here linear and dissipated, while the SDE for $\bar{H} \bar{a}$ acts as an external source of perturbations that sustains ENSO variability. Note that the probability density function (pdf) of $T_{E}$ is nearly Gaussian, while the pdf of $\bar{H} \bar{a}$ matches the theoretical gamma distribution from Fig. 2d (not shown).

Figures $3 \mathrm{c}-\mathrm{g}$ show the details of an El Niño event (around year 1623) with strong SST anomalies representative of extreme events in the observational record (e.g., 1997/98, 2015/16). The event starts with a realistic build up of SST and thermocline depth anomalies in the western Pacific that eventually propagate and intensify in the eastern Pacific. Zonal wind anomalies $\bar{u}$ become positive in the western to central Pacific consistent with the gradual weakening of the trade winds. The El Niño event is then followed by a reversal of conditions the following year toward a weak La Niña state.

\section{b. Crude intraseasonal model}

We now show solutions of the crude intraseasonal model [see Fig. 1c and Eqs. (3), (5)-(7), (11) for its formulation]. In the crude intraseasonal model, the intraseasonal atmosphere dynamics are solved explicitly. Important nonlinear and multiplicative noise features of convection are, however, missing that will be accounted for hereafter with the complete TSS-GCM (Majda and Stechmann 2009; Thual et al. 2014; Chen and Majda 2016a). Another caveat of the crude intraseasonal model is the presence of unrealistic excessive westward propagations in the atmosphere.

Figures $4 \mathrm{a}, 4 \mathrm{~b}, 4 \mathrm{~d}$, and $4 \mathrm{e}$ show the power spectra of the intraseasonal atmosphere variables as a function of 
(a) $u^{\prime}$
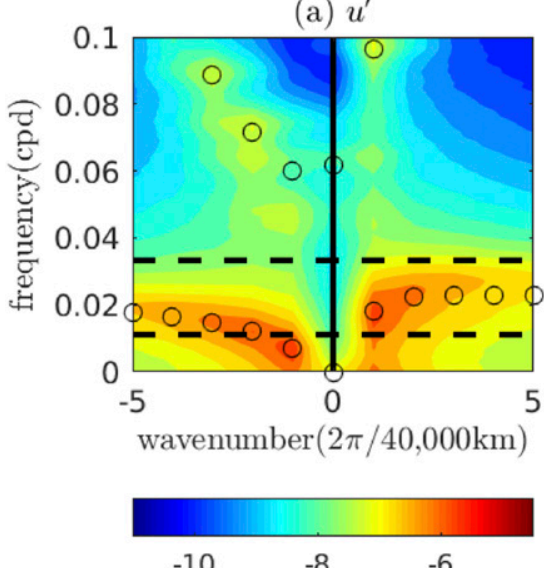

(d) $\theta^{\prime}$
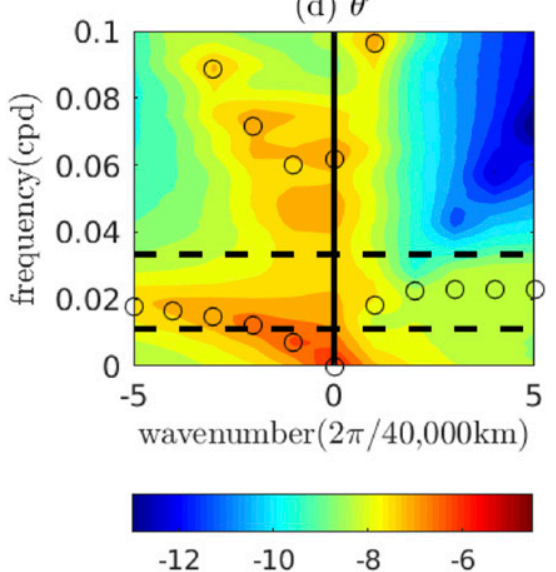

(b) $a^{\prime}$
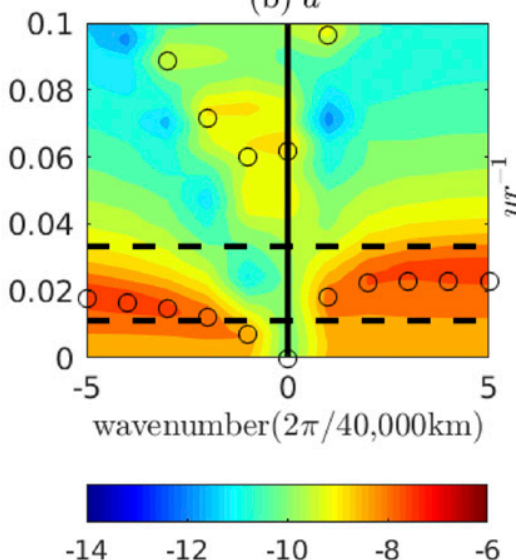

(e) $q^{\prime}$

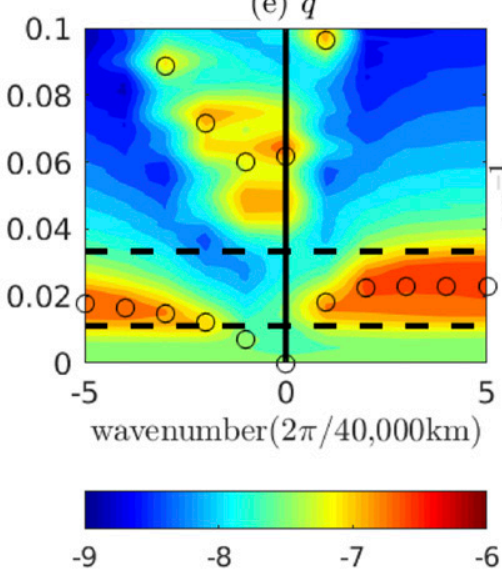

(c) $u_{W}^{\prime}$

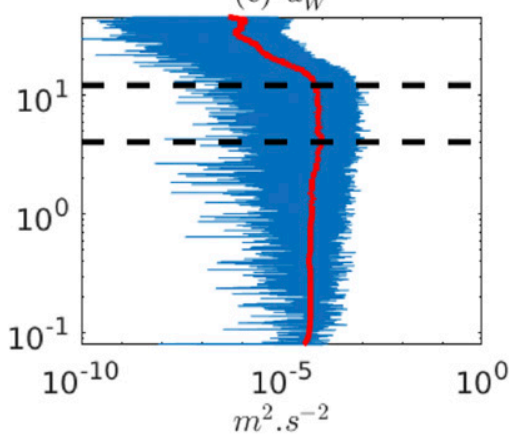

(f) $T_{E}$

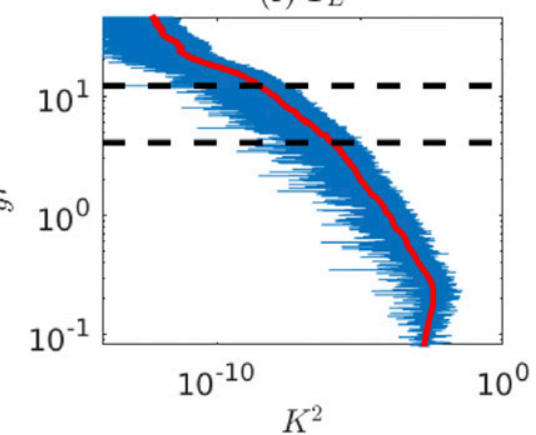

FIG. 4. Solutions of the crude intraseasonal model. Zonal wavenumber-frequency power spectra for intraseasonal (a) zonal winds $u^{\prime}\left(\mathrm{m} \mathrm{s}^{-1}\right),(\mathrm{b})$ convective activity $\bar{H} a^{\prime}\left(\mathrm{K}_{\text {day }}{ }^{-1}\right),(\mathrm{d})$ potential temperature $\theta^{\prime}(\mathrm{K})$, and (e) moisture $q^{\prime}(\mathrm{K})$ as a function of wavenumber $(2 \pi / 40000 \mathrm{~km})$ and frequency (cpd). The contour levels are in the base-10 logarithm for the dimensional variables taken at the equator. The dots indicate the dispersion relations of the linearized intraseasonal atmosphere. Power spectrum of (c) $u_{W}^{\prime}$ the average of $u^{\prime}$ at the equator in the western half of the equatorial Pacific (blue; $\mathrm{m} \mathrm{s}^{-1}$ ) and (f) $T_{E}$ the average of $T$ at the equator in the eastern half (blue; K), in addition to their smoothed versions (red). The dashed line indicates the periods 30 and 90 days in all subplots.

the zonal wavenumber $k(2 \pi / 40000 \mathrm{~km})$ and frequency $\omega(\mathrm{cpd})$. The intraseasonal atmosphere reproduces an MJO-like signal that is the dominant intraseasonal signal, consistent with observations [see, e.g., Fig. 3b of Wheeler and Kiladis (1999) for comparison; Thual et al. 2014; Stechmann and Hottovy 2017]. The MJO appears here as a sharp power peak in the intraseasonal planetary band $(1 \leq k \leq 5$ and $1 / 90 \leq \omega \leq 1 / 30 \mathrm{cpd})$, most prominent in $u^{\prime}, q^{\prime}$, and $\bar{H} a^{\prime}$. This power peak roughly corresponds to the slow eastward phase speed of $\omega / k \approx 5 \mathrm{~m} \mathrm{~s}^{-1}$ with the peculiar relation dispersion $d \omega / d k \approx 0$ found in observations. There is, however, excessive westward power in the intraseasonal band $(-3 \leq k \leq-1$ and $1 / 90 \leq \omega \leq 1 / 30 \mathrm{cpd})$ as seen for $\theta^{\prime}, q^{\prime}$, and $a^{\prime}$, which is a caveat of the present crude intraseasonal model. Note that power is maximal near the dispersion curves of the linear solutions of the intraseasonal atmosphere [black dots; see Thual et al. (2014) for a discussion].

To understand the time scale interaction between El Niño and the wind bursts, Figs. $4 \mathrm{c}$ and $4 \mathrm{f}$ show the power spectrum of $T_{E}$ the average of SSTs in the eastern Pacific, as well as $u_{W}^{\prime}$ the average of intraseasonal winds in the western Pacific half. The index $T_{E}$ is here a good indicator of ENSO variability in the model, while the index $u_{W}^{\prime}$ is a good indicator of the wind bursts' variability. Both power spectra are shown in log-log scale to cover both the interannual and intraseasonal range, and the dashed lines indicate the intraseasonal 30-90-day band from Figs. 4a, 4b, 4d, and 4e. First, the spectrum of $u_{W}^{\prime}$ is approximately white (with power evenly distributed) except for fluctuations below 30 days that are 

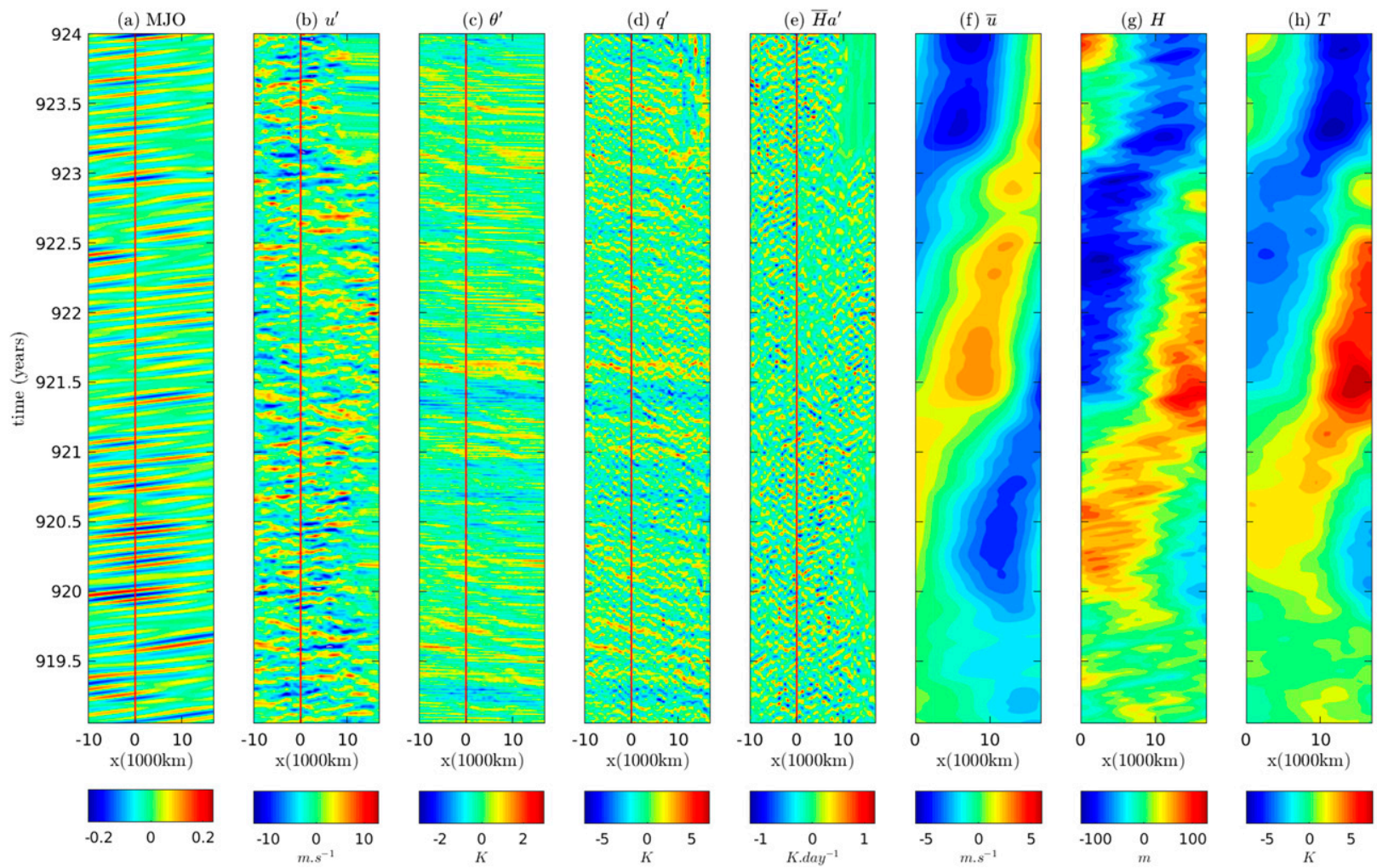

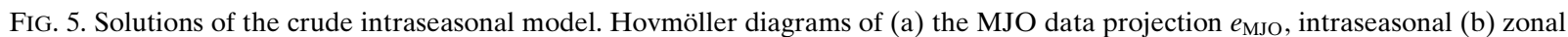
winds $u^{\prime}\left(\mathrm{m} \mathrm{s}^{-1}\right)$, (c) potential temperature $\theta^{\prime}(\mathrm{K})$, and (d) moisture $q^{\prime}(\mathrm{K})$, as well as (e) interannual zonal winds $\bar{u}\left(\mathrm{~m} \mathrm{~s}^{-1}\right)$, (f) thermocline depth $H(\mathrm{~m})$, and $(\mathrm{g})$ SST $T(\mathrm{~K})$ at the equator, as a function of zonal position $x(1000 \mathrm{~km})$ and time (years). Red line indicates the western Pacific edge at $x=0$. The Hovmöller diagrams in (a)-(e) extend from -10000 to $18000 \mathrm{~km}$ (Indian and Pacific Oceans), while in (f)-(h) they extend from 0 to $18000 \mathrm{~km}$ (Pacific Ocean only).

dissipated. Associated with this, the spectrum of $T_{E}$ is approximately red (i.e., decreasing linearly with frequency) consistent with the time integration of wind bursts' noise by the interannual ocean and atmosphere. Second, the spectrum of $T_{E}$ shows a peak at around $0.2 \mathrm{yr}^{-1}(\approx 4.5 \mathrm{yr})$, consistent with the average ENSO period found in the linear solutions of the interannual atmosphere and ocean (Thual et al. 2016). Note in particular that intraseasonal variability in the 30-90-day band is clearly separated from the average ENSO period.

Figure 5 shows the details of intraseasonal variability during a strong El Niño event (around year 922). Consistent with the model formulation, the intraseasonal atmosphere evolves on a different time scale than the interannual atmosphere and ocean, with the exception of some intraseasonal disturbances on thermocline depth that correspond mainly to eastward-propagating ocean Kelvin waves. Figure 5a shows a data projection $e_{\mathrm{MJO}}$ that evaluates the MJO intensity by comparison to the linear solutions of the crude intraseasonal atmosphere. Such a data projection is obtained by filtering the intraseasonal atmosphere signals in the intraseasonal planetary band $(1 \leq k \leq 3,1 / 90 \leq \omega \leq 1 / 30 \mathrm{cpd})$, then projecting them on the MJO linear solution eigenvector (see Majda and Stechmann 2011; Thual et al. 2014; Stechmann and Majda 2015 for details). This representation, along with the other Hovmöller diagrams shown in Fig. 5, allows us to identify clearly the MJO variability despite the noisy signals. On average, the simulated MJO events propagate eastward with a phase speed $\approx$ $5-15 \mathrm{~m} \mathrm{~s}^{-1}$ and period $\approx 40$ days and are furthermore organized into wave trains (i.e., series) of successive events, as in nature.

The El Niño event onset in Fig. 5 (around year 920-922) consists of a build up of SST and thermocline depth anomalies starting from the western Pacific. During the event onset, intraseasonal wind bursts $u^{\prime}$, convective activity $\bar{H} a^{\prime}$, and the MJO gradually intensify and expand toward the central to eastern Pacific, as in nature (Eisenman et al. 2005; Hendon et al. 2007; Tziperman and Yu. 2007; Gebbie et al. 2007). Some MJO wave trains even reach the eastern Pacific during the event peak (around year 922). Note that in the absence of El Niño events, intraseasonal variability remains 
(a) MJO

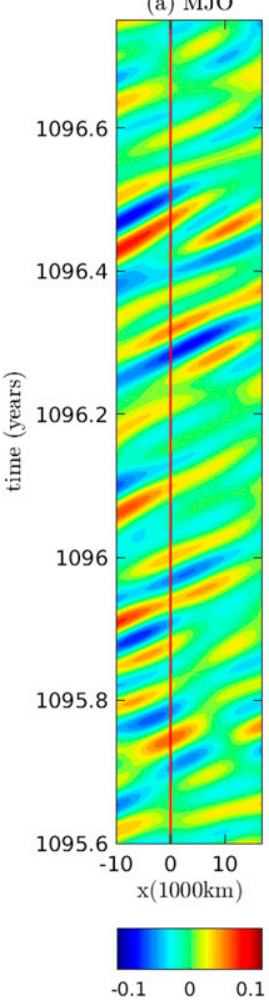

(b) $u^{\prime}$

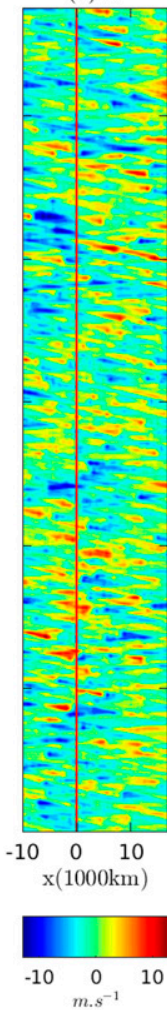

(c) $\bar{u}+u^{\prime}$

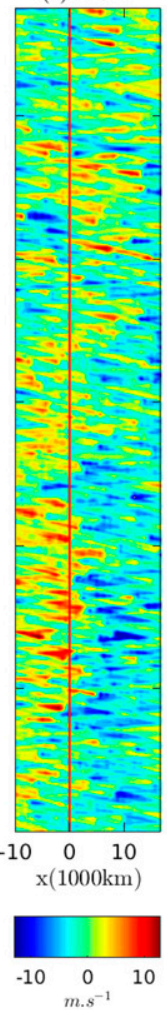

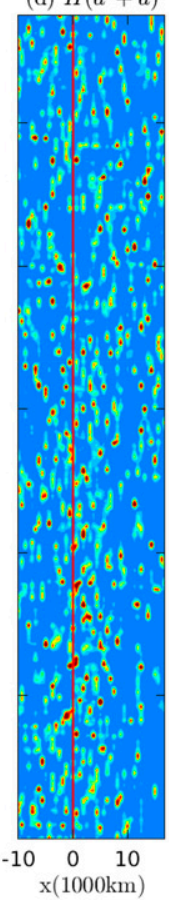

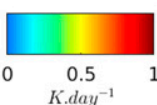

(e) $\bar{H} \bar{a}$

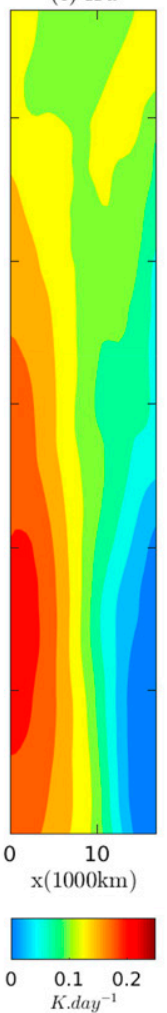

(f) $\bar{u}$

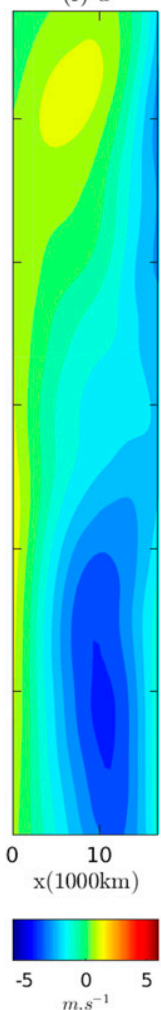

(g) $H$

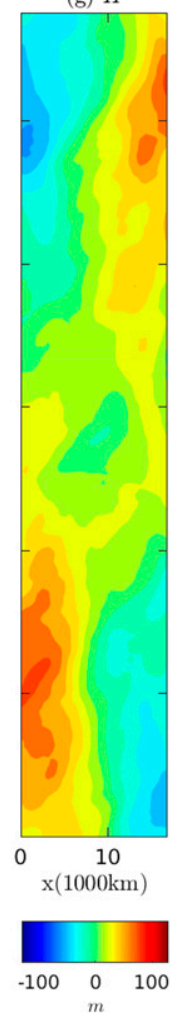

(h) $T$

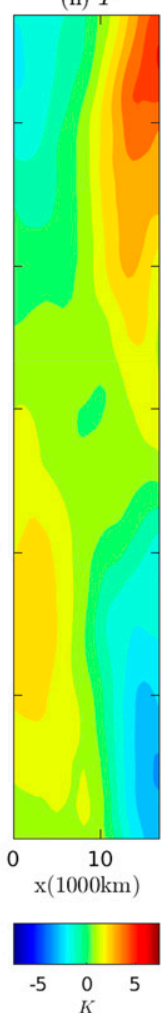

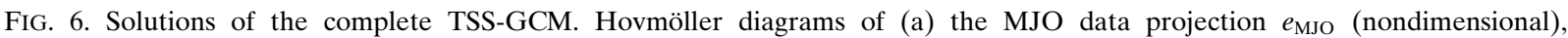
(b) intraseasonal zonal winds $u^{\prime}\left(\mathrm{m} \mathrm{s}^{-1}\right)$, (c) total zonal winds $\bar{u}+u^{\prime}\left(\mathrm{m} \mathrm{s}^{-1}\right)$, (d) total convective activity $\bar{H}\left(\bar{a}+a^{\prime}\right)\left(\mathrm{K}_{\text {day }}{ }^{-1}\right.$ with values above $1 \mathrm{~K} \mathrm{day}^{-1}$ not contoured), as well as (e) interannual convective activity $\bar{H} \bar{a}\left(\mathrm{~K} \mathrm{day}^{-1}\right)$, (f) interannual zonal winds $\bar{u}\left(\mathrm{~m} \mathrm{~s}{ }^{-1}\right)$, (f) thermocline depth $H(\mathrm{~m})$, and $(\mathrm{g})$ SST $T(\mathrm{~K})$ at the equator, as a function of zonal position $x(1000 \mathrm{~km})$ and time (years). The red line indicates the western Pacific edge at $x=0$. The Hovmöller diagrams in (a)-(e) extend from -10000 to $18000 \mathrm{~km}$ (Indian and Pacific Oceans), while in (f)-(h) they extend from 0 to $18000 \mathrm{~km}$ (Pacific Ocean only).

confined overall to the Indian Ocean and western $\mathrm{Pa}$ cific, consistent with the increased sources of cooling/ moistening $s^{\theta}$ and $s^{q}$ over that region (cf. Fig. 2a; see also Majda and Stechmann 2011). Finally, strong wind bursts or a prominent MJO do not necessarily trigger El Niño events (Fedorov et al. 2015; Hu et al. 2014), as shown, for example, with the strong wind bursts in Fig. 5 around year 919.5. Note in addition the presence of excessive westward propagations in Fig. 5 on wind bursts $u^{\prime}$, potential temperature $\theta^{\prime}$, and moisture $q^{\prime}$, which is a caveat of the present crude intraseasonal model.

\section{c. Complete TSS-GCM}

We now show the solutions of the complete TSSGCM [see Fig. 1a and Eqs. (3), (5)-(7), (12) for its formulation]. Such a model retains all the dynamics from the starting deterministic ocean and atmosphere and elements from the crude interannual and intraseasonal model versions presented above in addition to fundamental convective nonlinearities and associated suitable stochastic parameterizations. This allows the complete
TSS-GCM to capture realistically some important features of wind bursts in nature such as their sharpness and intensity in addition to ensuring a positive convective activity. For completeness, several diagnostics presented above for the crude interannual and intraseasonal model versions are repeated here for the complete TSS-GCM.

Figure 6 shows the details of a super El Niño event (around year 1096.8) simulated by the complete TSSGCM. Importantly, there are here more realistic intraseasonal and convective features as compared to the crude intraseasonal model (Fig. 5). This includes localized wind bursts ( $u^{\prime}$ in Fig. $\left.6 \mathrm{c}\right)$ in the western Pacific, both easterly or westerly, with a short lifespan around 10-30 days, sharp structure in both space and time, and large zonal fetch. Those wind bursts result from strong and localized peaks in convective activity $\left[\bar{H}\left(\bar{a}+a^{\prime}\right)\right.$ in Fig. 6d] as representative of deep convective events in nature, with heating reaching $1 \mathrm{~K}$ day $^{-1}$ or more, while convection is otherwise suppressed overall $\left(\approx 0.1 \mathrm{Kday}^{-1}\right)$. Such a realistic bursting behavior in both convection and wind bursts results from the parameterization of convection in 
Eq. (12) with non-Gaussian noise and fundamental nonlinearities. In addition to this, the complete TSS-GCM captures the eastward expansion of the sharp wind bursts and convective events during the onset of the El Niño event (Eisenman et al. 2005; Hendon et al. 2007; Tziperman and Yu. 2007; Gebbie et al. 2007). This is best seen in Fig. $6 \mathrm{c}$ on total zonal winds $\bar{u}+u^{\prime}$, for which westerly wind bursts are dominant in the western Pacific/ Indian Ocean at the event onset (1095.8-1096 yr) then gradually expand toward the eastern Pacific until the event peak (around $1096.8 \mathrm{yr}$ ). Those are all important and realistic features captured in a simple fashion by the complete TSS-GCM. Note that the total convective activity $\bar{a}+a^{\prime}$ remains positive in Fig. $6 \mathrm{~d}$, which is in agreement with the design principles for the model's atmosphere [Eqs. (1), (12)].

Figure 7 shows time series and Hovmöller diagrams for the interannual variability simulated by the complete TSS-GCM. The model simulates a sustained and irregular ENSO cycle with intermittent El Niño and La Niña events of varying intensity and strength, as in nature [see, e.g., Fig. 2 of Chen et al. (2018) for a comparison with observations]. Note, however, that in contrast to observations (e.g., Santoso et al. 2017), there are no central Pacific El Niños and that El Niño and La Niña events have an overly symmetric structure of eastwardpropagating SST anomalies peaking in the eastern Pacific. This discrepancy is due notably to the oversimplified SST thermodynamics in the model (Chen and Majda 2016b, 2017). In Fig. 7, there are two super El Niño events (around years 1606 and 1627) with strong SST anomalies as representative of extreme events in the observational record. The events are spaced by around 20 years, which happens to be similar to the waiting time between the 1997/98 and 2015/16 events in nature, though there are no other similar instances along the present 2000-yr integration. There are in addition many examples of moderate or failed El Niño events in Fig. 7. Figure $7 \mathrm{c}$ shows a 1 -yr running mean of $\left|e_{\mathrm{MJO}}\right|$ the magnitude of the data projection $e_{\mathrm{MJO}}$. This allows us to evaluate the interannual variations of the MJO intensity. The interannual variations of the MJO intensity are random overall, as resulting from the internal variability of the intraseasonal atmosphere alone [see, e.g., Fig. 5 of Thual et al. (2014) for comparison], though they are here modulated to some extent by the SSTs. For instance, the MJO intensity in Fig. 7c is increased from the western to eastern Pacific during several El Niño events.

Figure 8 shows lagged regressions of several interannual and intraseasonal variables on $T_{E}$ the average of SST in the Pacific eastern half. This highlights the overall formation mechanisms and chronology of $\mathrm{El}$
Niño events in the model [see, e.g., Fig. 7 of Chen et al. (2018) for a comparison with observations]. To identify the evolution of the intraseasonal atmosphere evolution during El Niño, we consider lagged regressions for the data projection $e_{\mathrm{MJO}}$ (cf. Fig. 6) and its magnitude $\left|e_{\mathrm{MJO}}\right|$, in addition to intraseasonal zonal winds $u^{\prime}$ and their magnitude $\left|u^{\prime}\right|$. As shown in Fig. 8, El Niño events typically start with increased thermocline depth and SST anomalies in the western Pacific that eventually propagate to the eastern Pacific, in addition to gradually increasing interannual winds. Those features are overall consistent with the Hovmöller diagrams in Fig. 7. Interestingly, the magnitude of intraseasonal variability in general ( $\left|e_{\mathrm{MJO}}\right|$ and $\left|u^{\prime}\right|$ in Figs. 8b,d) is increased overall in the western Pacific during the onset of El Niño as well as in the central to eastern Pacific during the event peak, as in nature (Vecchi and Harrison 2000; Hendon et al. 1999). This results from the increased SSTs that favor the temporal growth/decay of intraseasonal convective activity $a^{\prime}$ [cf. Eq. (12)]. Recall that wind bursts from the intraseasonal atmosphere trigger El Niño by design in the TSS-GCM because they are coupled to an interannual atmosphere that is otherwise stable, linear, and dissipated [Eqs. (3), (5)-(7)]. In fact, wind bursts $u^{\prime}$ in Fig. 8c are predominantly westerly in the western to central Pacific around six months prior to the event peak. This forces a deepening of the equatorial thermocline in the ocean (i.e., downwelling equatorialocean Kelvin and Rossby waves) that contributes to the increase of SSTs in the east and triggers the El Niño event (Hu and Fedorov 2017). Interestingly, the location and timing of those predominantly westerly wind bursts in Fig. $8 \mathrm{c}$ does not match the one of the overall increased magnitude ( $\left|u^{\prime}\right|$ in Fig. 8d), suggesting that only some wind bursts may be key for El Niño triggering. On the other hand, lagged regressions are weak for the MJO variability $\left(e_{\mathrm{MJO}}\right.$ in Fig. 8a) despite an increased magnitude $\left|e_{\mathrm{MJO}}\right|$. In fact, the MJO approximately oscillates at a period of $\approx 40$ days, which has opposite and canceling effects on the ocean that are ineffective at triggering El Niño events. The lagged regressions of $e_{\mathrm{MJO}}$ are, however, increased during the event peak as a result of the increased (decreased) SSTs and associated convection in the eastern (western) Pacific.

Finally, Fig. 9 documents the power spectra and statistical features of the complete TSS-GCM. First, Figs. 9a, 9b, 9e, and 9f show the power spectra for variables of the intraseasonal atmosphere. While the features are overall similar to the ones of the crude intraseasonal model version (Fig. 4), there are here fewer westward propagations in the intraseasonal 30 90-day band as seen for $u^{\prime}$ as well as $q^{\prime}$ and $\theta^{\prime}$, which is more realistic. Second, Figs. $9 \mathrm{c}$ and $9 \mathrm{~g}$ show the power 
(a) $T_{E}$
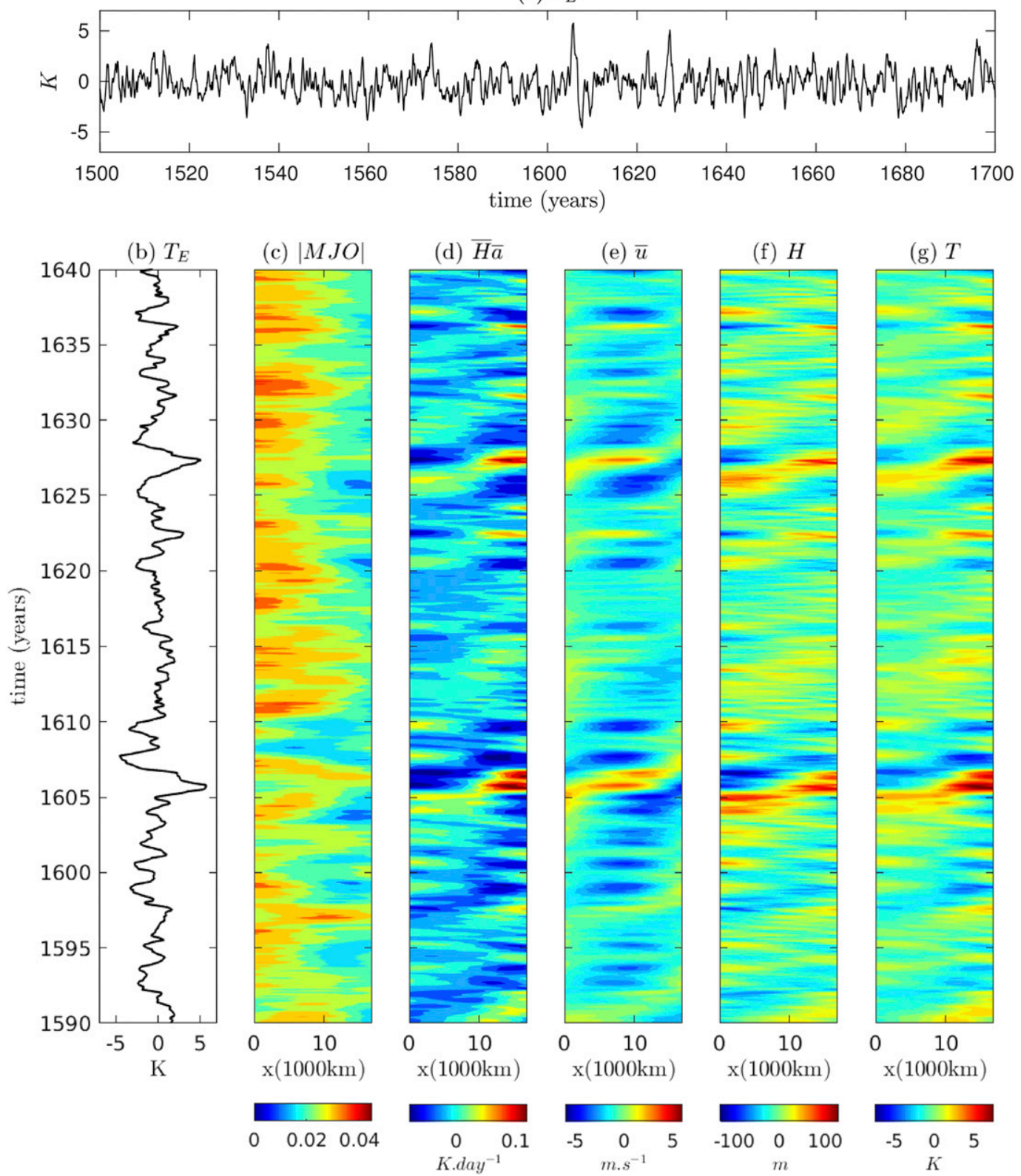

(e) $\bar{u}$

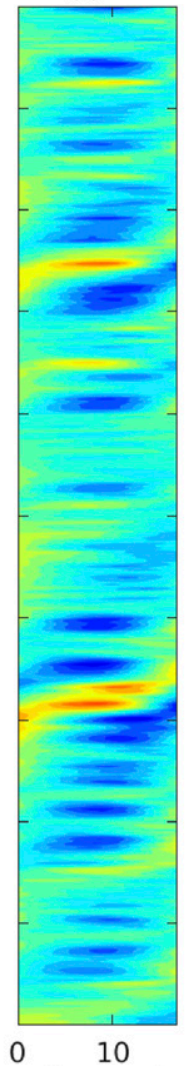

$\mathrm{x}(1000 \mathrm{~km})$

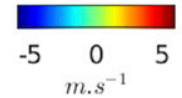

(f) $H$

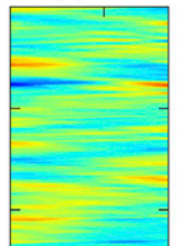

(g) $T$

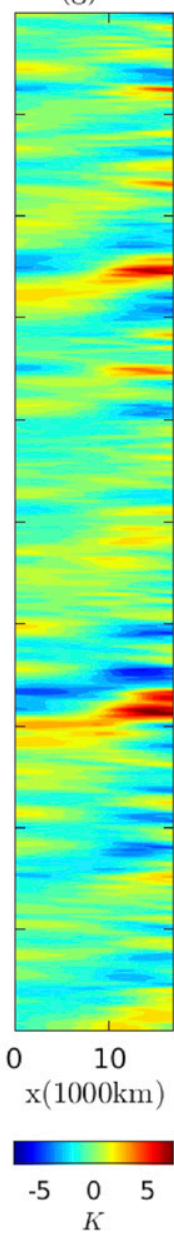

FIG. 7. Solutions of the complete TSS-GCM. Time series of (a) $T_{E}$ the average of SSTs at the equator in the eastern half of Pacific (K). (b) Repeats of the time series over a shorter period. Hovmöller diagrams of (c) a 1-yr running mean of $\left|e_{\mathrm{MJO}}\right|$ the magnitude of the MJO data projection $e_{\mathrm{MJO}}$, (d) interannual convective activity $\bar{H} \bar{a}\left(\mathrm{~K} \mathrm{day}^{-1}\right)$, (e) zonal winds $\bar{u}\left(\mathrm{~m} \mathrm{~s}^{-1}\right)$, (f) thermocline depth $H(\mathrm{~m})$, and (g) SST $T(\mathrm{~K})$ at the equator, as a function of zonal position and time (years).

spectrum of $T_{E}$ the average of SSTs in the eastern Pacific, as well as the power spectrum of $u_{W}^{\prime}$ the average of intraseasonal wind bursts in the western Pacific half. As compared to the crude interannual atmosphere model version (Fig. 4), the spectrum of $u_{W}^{\prime}$ shows a slight peak around $0.2 \mathrm{yr}^{-1}(\approx 4.5 \mathrm{yr})$ corresponding to the average ENSO period in the model. This suggests that wind bursts' variability is modulated interannually to some extent by ENSO SSTs consistent with the lagged regressions in Fig. 8d. Figure 9d shows the pdf of total convective activity $\bar{H}\left(a^{\prime}+\bar{a}\right)$ at the Pacific western edge. The pdf matches to some extent the theoretical gamma distribution from Eq. (10) and Fig. 2d (which ensures notably that $a^{\prime}+\bar{a}$ remains positive), though it is significantly more skewed toward extreme convective events because of the addition of deterministic convective nonlinearities 

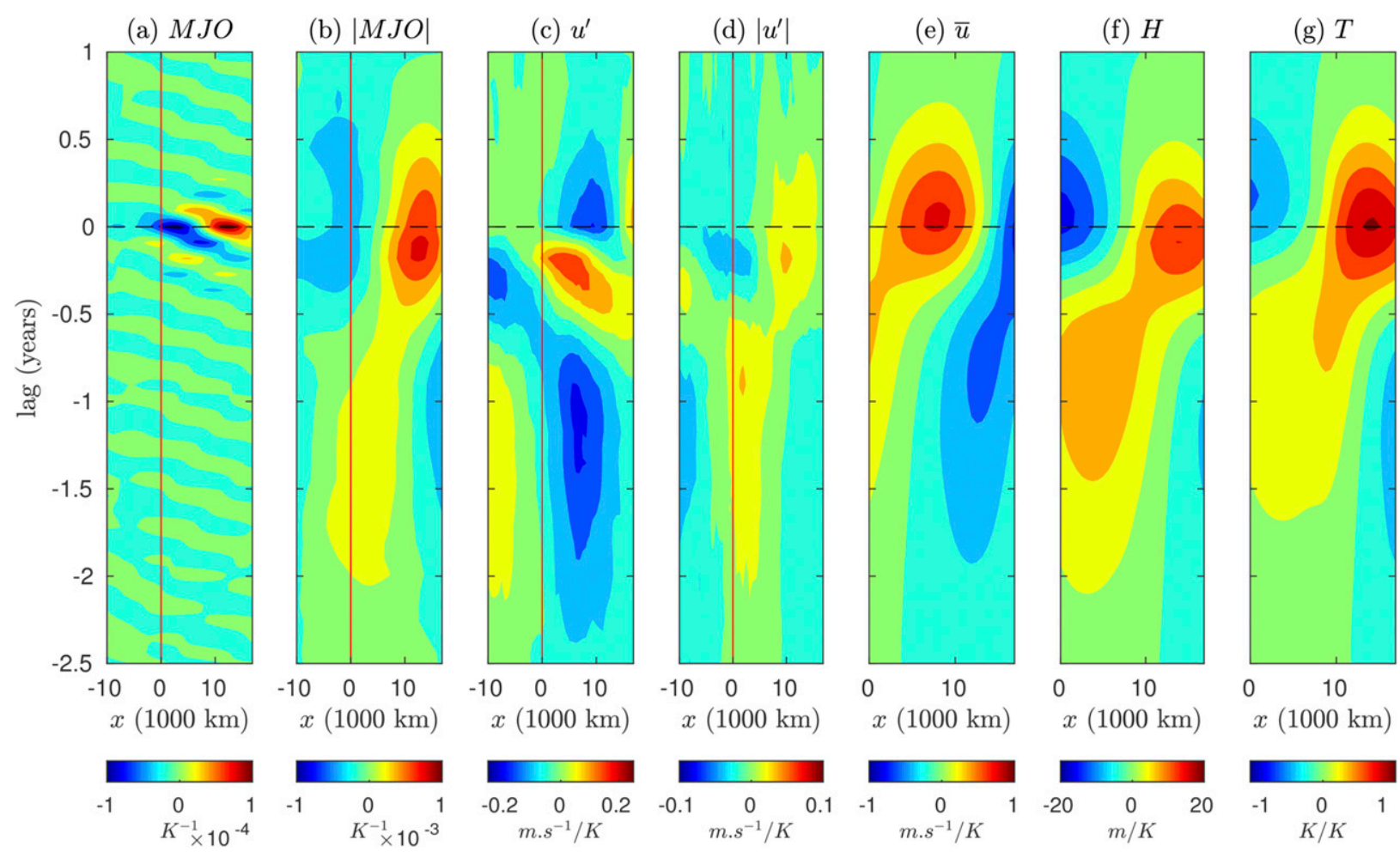

FIG. 8. Solutions of the complete TSS-GCM. Lagged regressions on $T_{E}$ (the average of SST at the equator in the eastern half of Pacific) of (a) the MJO data projection $e_{\mathrm{MJO}}$ and (b) its magnitude $\left|e_{\mathrm{MJO}}\right|\left(\mathrm{K}^{-1}\right)$, (c) intraseasonal winds $u^{\prime}$, and (d) their magnitude $\left|u^{\prime}\right|$ $\left(\mathrm{m} \mathrm{s}^{-1} \mathrm{~K}^{-1}\right)$, (e) interannual winds $\bar{u}\left(\mathrm{~m} \mathrm{~s}^{-1} \mathrm{~K}^{-1}\right)$, (f) thermocline depth $H\left(\mathrm{~m} \mathrm{~K}^{-1}\right)$ and (g) SST $T$ (nondimensional) at the equator, as a function of zonal position $x(1000 \mathrm{~km})$ and lag (years; positive for $T_{E}$ leading). The red line indicates the western Pacific edge at $x=0$.

in the complete TSS-GCM $\left[\Gamma q^{\prime}\left(\bar{a}+a^{\prime}\right)\right]$ in Eq. (12). Finally, Fig. $9 \mathrm{~h}$ shows the pdfs for $T_{E}$ as well as for the observed index Niño-3 SST [computed from the Optimum Interpolation Sea Surface Temperature (OISST) reanalysis; Kalnay et al. 1996]. The pdf of Niño-3 SST is skewed as representative of rare extreme El Niño events in nature (Jin et al. 2007; Thual et al. 2016). The pdf of $T_{E}$ in contrast is nearly Gaussian, which is a limitation of the present model version (see discussion section hereafter). The amplitude of $T_{E}$ is also slightly too strong as compared to observations, though this could be more easily corrected by tuning, for example, the intraseasonal noise intensity in the model.

\section{d. Complete TSS-GCM with dynamic Walker circulation}

We now show solutions of the TSS-GCM with dynamic Walker circulation. Such a model version is identical to the TSS-GCM presented above except for the introduction of unbalanced external sources of cooling/moistening $s^{\theta} \neq s^{q}$ (Fig. 2b). Note that a dynamic Walker circulation can be obtained for $s^{\theta} \neq s^{q}$ in any version of the TSS-GCM (crude interannual, crude intraseasonal, or complete TSS-GCM).
Figure 10 shows the background mean (i.e., climatological) circulation, obtained from a time average of the model solutions. The equilibrium atmosphere is marked by mean westward trade winds and an overturning circulation in the upper troposphere. It consists of a region of ascent, convergence, and increased convection in the western Pacific as well as subsidence and divergence in the eastern Pacific. Those are all realistic features representative of the Walker circulation in nature (Ogrosky and Stechmann 2015). Note that the present atmosphere has a first baroclinic mode structure, with reconstruction $\bar{u}=\bar{u}(x) \cos (z)$ as well as $\bar{w}=-\partial_{x} \bar{u} \sin (z)$ (see, e.g., Chen and Majda 2016b). Meanwhile, the equilibrium atmospheric circulation maintains zonal gradients of SST $(\approx 8 \mathrm{~K})$ and thermocline depth $(\approx 200 \mathrm{~m})$ in the ocean, whose intensities compare reasonably with the ones found in nature (Clarke 2008). Finally, other features of the present model version are overall similar to the ones of the complete TSS-GCM (Figs. 9-7) and are not shown for brevity.

\section{Discussion}

In the present article, a simple dynamical stochastic model for ENSO, MJO, and intraseasonal variability in 

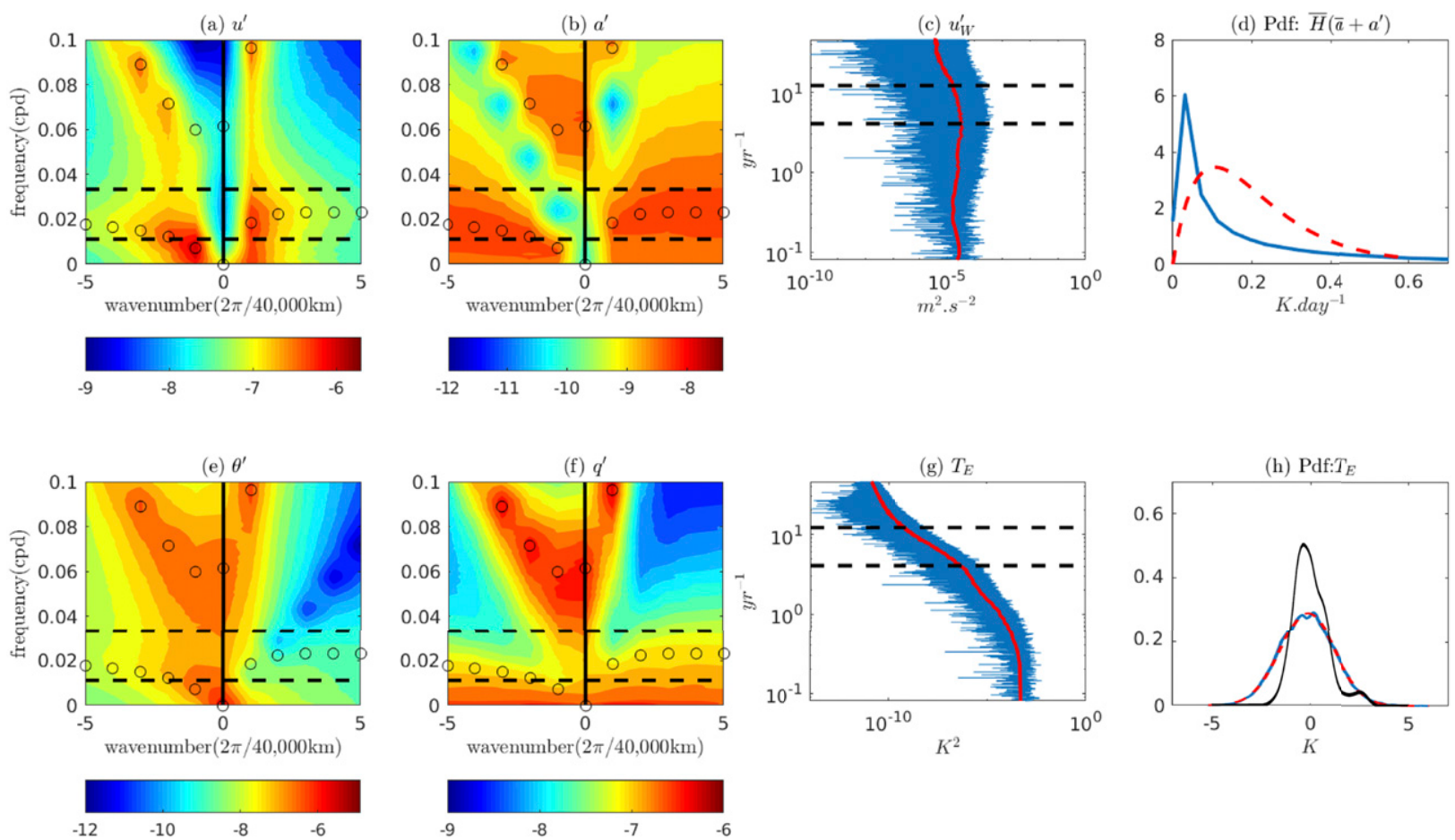

FIG. 9. Solutions of the complete TSS-GCM. Zonal wavenumber-frequency power spectra for intraseasonal (a) zonal winds $u^{\prime}\left(\mathrm{m} \mathrm{s}{ }^{-1}\right)$, (b) convective activity $a^{\prime}\left(\mathrm{K}_{\text {day }}{ }^{-1}\right)$, (e) potential temperature $\theta^{\prime}(\mathrm{K})$, and (f) moisture $q^{\prime}(\mathrm{K})$, as a function of wavenumber $(2 \pi / 40000 \mathrm{~km})$ and frequency (cpd). The contour levels are in the base-10 logarithm for the dimensional variables taken at the equator. The dots indicate the dispersion relations of the linearized intraseasonal atmosphere. Power spectrum of (c) $u_{W}^{\prime}$ the average of $u^{\prime}$ at the equator in the western half of the equatorial Pacific $\left(\mathrm{m} \mathrm{s}^{-1}\right)$ and $(\mathrm{g})$ of $T_{E}$ the average of $T$ at the equator in the eastern half $(\mathrm{K})$. The dashed black lines indicate the periods 30 and 90 days in all subplots. (d) The pdf of total convective activity $\bar{H}\left(\bar{a}+a^{\prime}\right)$ at the equator and at the warm pool center/western Pacific edge $x=0\left(\mathrm{~K} \mathrm{day}^{-1}\right)$. The red dashed line in (d) indicates the corresponding equilibrium gamma distribution from Eq. (10) for $E_{q}=0$. (h) The pdf of $T_{E}(\mathrm{~K}$; blue) as well as a Gaussian fit (red dashed). The black line in (h) is the pdf of Niño-3 SST in observations.

general as well as the dynamic Walker circulation has been introduced and developed in detail. The present model, the TSS-GCM, serves as a prototype for GCMs that solve similar dynamical interactions across several spatiotemporal scales but usually show common and systematic biases in representing tropical variability as a whole. The present model formulation builds on previous work by the authors, namely, a simple deterministic ocean-atmosphere for ENSO (Thual et al. 2016, 2017; Chen and Majda 2016b, 2017, 2018) in addition to a skeleton model for the MJO and intraseasonal variability in general (Majda and Stechmann 2009, 2011; Thual et al. 2014). In particular, a simple decomposition of the atmospheric flow in the present TSS-GCM allows us to represent in simple fashion both the interannual and intraseasonal dynamics as well as their interactions. The most salient features of ENSO, wind bursts, and the MJO are captured altogether including their overall structure, evolution, and energy distribution across scales, in addition to their intermittency and diversity as well as their fundamental interactions. For instance, El Niño events in the model are realistically triggered by increased and predominantly westerly wind bursts starting in the Indian to western Pacific Oceans and expanding eastward toward the central Pacific (Vecchi and Harrison 2000; Fedorov et al. 2015). In return, the characteristics of wind bursts and the MJO are significantly modulated interannually by ENSO SSTs, as in nature (Zhang and Gottschalck 2002; Hendon et al. 2007). These are attractive features of the present dynamical stochastic model.

As compared to former simple models dealing with ENSO that usually consider stochastic wind bursts (e.g., Moore and Kleeman 1999; Eisenman et al. 2005; Jin et al. 2007; Chen et al. 2015; Thual et al. 2016), the present model has the advantage of featuring intraseasonal wind bursts that are dynamically solved. For instance, there is no arbitrary prescription of wind bursts' amplitudes, propagations, or abrupt convection thresholds. As a result, the wind bursts' upscale contributions to the interannual flow and their modulation in return are provided in a more explicit way. As compared 

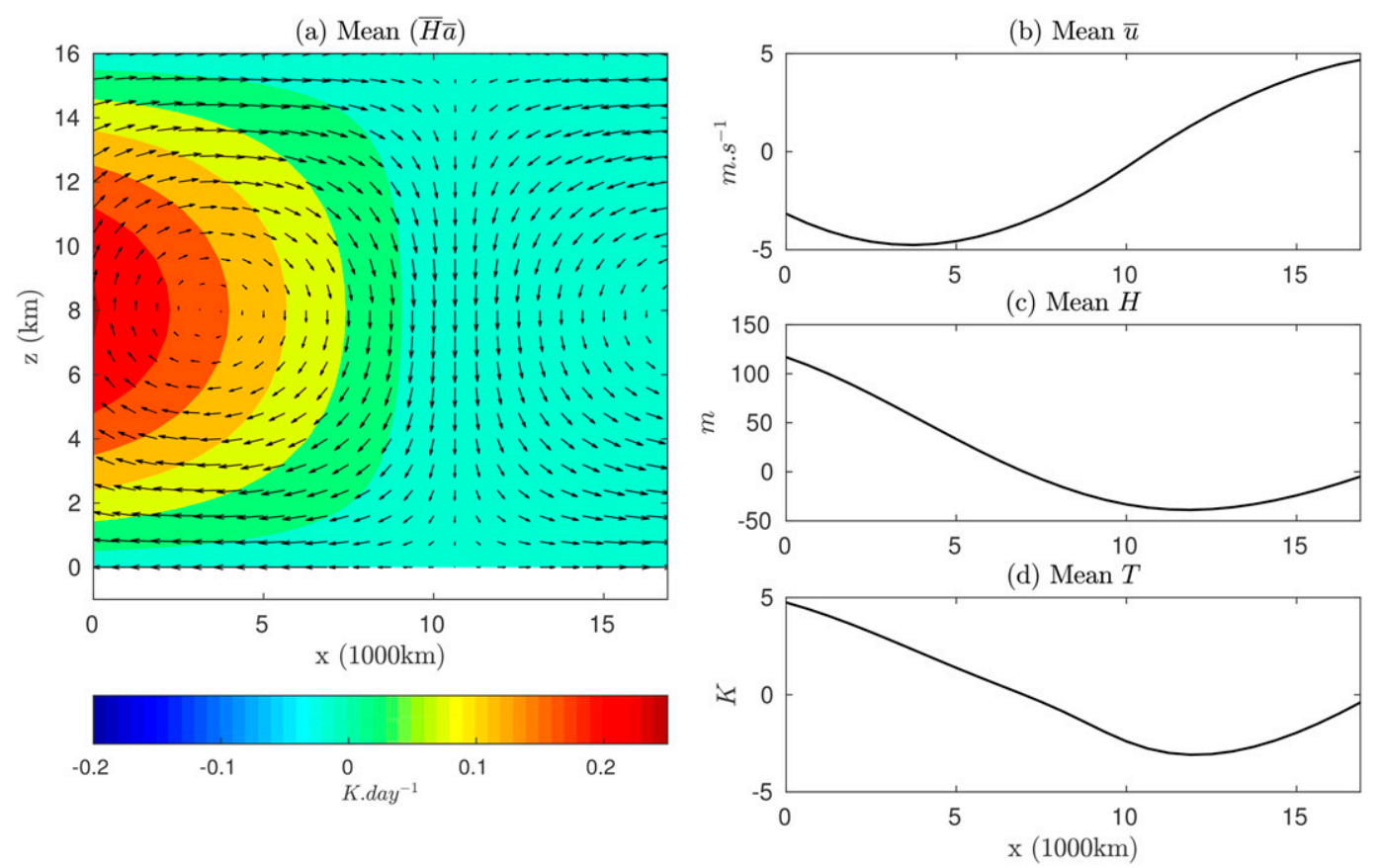

FIG. 10. Solutions of the TSS-GCM with dynamic Walker circulation. (a) Contours of time-averaged interannual convective activity $\bar{H} \bar{a}\left(\mathrm{~K} \mathrm{day}^{-1}\right)$ at the equator, as a function of zonal position $(1000 \mathrm{~km})$ and height $(\mathrm{km})$ in the equatorial Pacific. Arrows indicate time-averaged interannual zonal and vertical wind speed. Zonal profiles of timeaveraged (b) interannual zonal winds $\bar{u}\left(\mathrm{~m} \mathrm{~s}^{-1}\right)$, (c) thermocline depth $H(\mathrm{~m})$, and (d) SST $T(\mathrm{~K})$ at the equator.

to some intermediate models for ENSO that do solve intraseasonal dynamics (e.g., Neelin and Zeng 2000; Zeng et al. 2000; Gushchina and Dewitte 2011), the present model also has the advantage of involving simpler and more tractable intraseasonal as well as interannual dynamics. Despite this, some limitations of the present intraseasonal dynamics need to be addressed. For instance, while the skeleton model atmosphere used in the present study appears to give a plausible representation of the MJO essential mechanisms (Majda and Stechmann 2009, 2011), including the intermittent generation of wind bursts within the MJO convective envelope (Thual et al. 2014; Puy et al. 2016), because of its minimal design such a skeleton model atmosphere does not account for additional important processes that generate wind bursts such as tropical cyclones or extratropical cold surges, among others (Harrison and Vecchi 1997; Vecchi and Harrison 2000; Kiladis et al. 2009; Chen et al. 2016).

A more complete model should account for more details of the interannual ocean and atmosphere dynamics relevant to ENSO. In fact, earlier work from the authors has largely extended the ENSO dynamics of the present model in order to facilitate additional realistic features such as the occurrence of the central Pacific El Niño (Chen and Majda 2016b, 2017, 2018) or the delayed super El Niño as of 2015/16 (Thual et al. 2018) in addition to the synchronization of ENSO to the seasonal cycle (Thual et al. 2017), among others. Although such features have been omitted in the present article in order to focus on the inclusion of intraseasonal dynamics, it would be important to account for them in future work. Central Pacific El Niño events, for example, (Ashok et al. 2007) may be facilitated by the addition of nonlinear advection of SSTs in the model (Chen and Majda 2016b). Another important feature found in those earlier works are realistic non-Gaussian SST statistics as representative of rare extreme El Niño events (Thual et al. 2016; Chen et al. 2018), a feature that is not recovered in the present TSS-GCM (cf. Fig. 9h). Such a limitation is also common in GCMs and may be improved, for example, by rendering the stochastic noise in the TSS-GCM [Eq. (12)] more multiplicative or organized (Jin et al. 2007).

The TSS-GCM developed here should be useful to diagnose, analyze, and help eliminate the strong tropical biases that exist in current operational models. Generally speaking, GCMs typically show common and systematic biases in representing ENSO, the MJO, and background circulation altogether (Lin et al. 2006; Wittenberg et al. 2006; Kim et al. 2009). Those biases usually arise because GCMs solve a vast range of strongly interacting processes on many spatial and temporal scales. The TSS-GCM in comparison shows 
great skill at capturing qualitatively both intraseasonal and interannual processes. This provides theoretical insight on the essential dynamics and interactions of such processes, which is a main goal of the present work. In addition to addressing the model's current limitations as discussed above, another perspective for future work would be to compare in detail the TSS-GCM's interannual and intraseasonal variability with one of GCMs with common deficiencies. In addition to this, the TSS-GCM may be extended in order to analyze additional features of tropical variability commonly found or misrepresented in GCMs. This may include, for example, the analysis of tropical decadal variability and/or climate projections simulated by the model, in addition to the tropical/extratropical interactions as well as the atmospheric coupling between different tropical basins, among others (Illig and Dewitte 2006; Wittenberg et al. 2014; Guilyardi et al. 2016; Chen et al. 2016). The present TSS-GCM may provide novel theoretical insight on the above features thanks to its simple and tractable dynamics in addition to its low computational cost.

Acknowledgments. The research of A.J.M. is partially supported by the Office of Naval Research Grant ONR MURI N00014-12-1-0912 and the center for Prototype Climate Modeling at the NYU Abu Dhabi Research Institute. S.T. and N.C. are supported as postdoctoral fellows through A.J.M's ONR MURI Grant.

\section{APPENDIX A}

\section{Derivation of the Starting Deterministic Atmosphere}

This section details the derivation of the starting deterministic atmosphere used in the TSS-GCM from a multiple-time approach (Majda and Klein 2003). A general motivation for this is to derive approximate solutions for slowly varying fluctuations in the atmosphere. For this, we assume the Reynolds hypothesis that such slowly varying fluctuations exist on the interannual time $\tau$, in addition to fast varying fluctuations on the intraseasonal time $t$ with zero mean on the slow time. Assuming the Reynolds hypothesis, the starting atmosphere from Eq. (1) is decomposed as follows:

$$
\begin{aligned}
a(x, y, t) & =\bar{a}(x, y, \epsilon t)+a^{\prime}(x, y, \epsilon t, t), \quad \text { i.e. } \\
a(x, y, \tau, t) & =\bar{a}(x, y, \tau)+a^{\prime}(x, y, \tau, t),
\end{aligned}
$$

and similarly for $u, v, \theta$, and $q$, with the relation between time variables $\tau=\epsilon t$ where the Froude number $\epsilon$ is an asymptotically small parameter. SSTs in nature, however, show weak intraseasonal variability compared to the interannual one, therefore associated latent heat release decomposes as $E_{q}=\bar{E}_{q}+\epsilon E_{q}^{\prime}$. The Reynolds operator is defined here as follows:

$$
\bar{a}(x, y, \tau)=\frac{1}{\Delta \tau} \int_{\tau-\Delta \tau / 2}^{\tau+\Delta \tau / 2} a(x, y, \tau, t) d t,
$$

where $\Delta \tau$ is a characteristic averaging interannual time scale. Note that for $\Delta \tau=\Delta t / \epsilon, \epsilon \rightarrow 0$ with $\Delta t$ constant the above Reynolds operator is asymptotically akin to a Reynolds time-mean average as in standard turbulence theory. Such an operator has the well-known properties $\partial_{t} \bar{a}=0$ and $\overline{a^{\prime}}=0$ as well as $\partial_{t} a=\epsilon \partial_{\tau} \bar{a}+\left(\epsilon \partial_{\tau}+\partial_{t}\right) a^{\prime}$. Next, we further decompose the variables in Eq. (14) into powers of $\epsilon$ small; that is, $a=a_{0}+\epsilon a_{1}+O\left(\epsilon^{2}\right)$. Combined with the above Reynolds decomposition, this reads as follows:

$$
\begin{aligned}
a(x, y, \tau, t)= & \bar{a}_{0}(x, y, \tau)+a_{0}^{\prime}(x, y, \tau, t)+\epsilon \bar{a}_{1}(x, y, \tau, t) \\
& +\epsilon a_{1}^{\prime}(x, y, \tau, t)+O\left(\epsilon^{2}\right) .
\end{aligned}
$$

The crucial requirements needed to formally guarantee that the terms $a_{0}=\bar{a}_{0}+a_{0}^{\prime}$ that describe the leading-order behavior in Eq. (16) are the sublinear growth conditions for the next-order terms $a_{1}=\bar{a}_{1}+a_{1}^{\prime}$ :

$$
\lim _{\epsilon \rightarrow 0}\left(\frac{a_{1}(x, y, \tau, \tau / \epsilon)}{|\tau / \epsilon|+1}\right)=0 .
$$

To obtain the interannual atmosphere, we decompose the starting atmosphere from 1 according to Eq. (16) and retain the leading-order dynamics [of $O(1)$ ]. This reads as follows:

$$
\begin{aligned}
-y \bar{v}_{0}-\partial_{x} \bar{\theta}_{0} & =0, \\
y \bar{u}_{0}-\partial_{y} \bar{\theta}_{0} & =0, \\
-\left[\partial_{x}\left(\bar{u}_{0}+\partial_{y} \bar{v}_{0}\right)\right] & =\bar{H} \bar{a}_{0}-s^{\theta}, \\
\bar{Q}\left(\partial_{x} \bar{u}_{0}+\partial_{y} \bar{v}_{0}\right) & =-\bar{H} \bar{a}_{0}+s^{q}+\bar{E}_{q 0}, \\
0 & =\overline{q_{0}} \overline{a_{0}}+\overline{q_{0}^{\prime} a_{0}^{\prime}},
\end{aligned}
$$

where a simple closure $\overline{q_{0}^{\prime} a_{0}^{\prime}} \propto \overline{q_{0}}$ is considered for the upscale contribution, leading to $\overline{q_{0}}=0$. With this simple closure, we retrieve the interannual atmosphere from Eq. (3) in the main text. Finally, the intraseasonal atmosphere from Eq. (2) is obtained by subtracting Eq. (3) from Eq. (1), and the subscript notation $a_{0}$ is dropped for brevity.

\section{APPENDIX B}

\section{Technical Details}

We provide here some additional technical details on the TSS-GCM formulation and numerical solving 
TABLE B1. Model parameter definitions and nondimensional values.

\begin{tabular}{lc}
\hline \multicolumn{1}{c}{ Parameter } & Nondimensional value \\
\hline Ratio of ocean/atmosphere phase speed $c$ & 0.05 \\
Froude number $\epsilon$ & 0.1 \\
$c_{1}=c / \epsilon$ & 0.5 \\
Equatorial belt length $L_{A}$ & $8 / 3$ \\
Equatorial Pacific length $L_{O}$ & 1.2 \\
Convective heating rate factor $\bar{H}$ & 22 \\
Mean vertical moisture gradient $\bar{Q}$ & 0.9 \\
Convective growth/decay rate $\Gamma$ & 1.66 \\
Latent heating factor $\alpha_{q}$ & 0.2 \\
Wind stress coefficient $\gamma$ & 6.53 \\
Latent heating exchange coefficient $\zeta$ & 8.7 \\
Profile of thermocline feedback $\eta$ & $\eta(x)=1.5+\left\{0.5\right.$ tanh $\left.\left[7.5\left(x-L_{O} / 2\right)\right]\right\}$ \\
Atmosphere dissipations $d_{a}, d_{q}, d_{\theta}, \lambda$ & 0.11 \\
Moisture noise amplitude $\sigma_{q}$ & 0.4 \\
External moistening source $s^{q}$ & $s^{q}=2.2\left[1+0.6 \cos \left(2 \pi x / L_{A}\right)\right]$ \\
External cooling source $s^{\theta}$ & $s^{\theta}=s^{q}$ except Walker circulation: \\
& $s^{\theta}=2.2\left[1+0.6 \cos \left(2 \pi x / L_{A}-0.1\right)\right]$ \\
\hline
\end{tabular}

algorithm. As regards the atmosphere and ocean domains, the atmosphere extends over the entire equatorial belt $0 \leq x \leq L_{A}$ with periodic boundary conditions $u(0, y, t)=u\left(L_{A}, y, t\right)$, etc., while the Pacific Ocean extends from $0 \leq x \leq L_{O}$ with reflection boundary conditions $\int_{-\infty}^{+\infty} U(0, y, t) d y=0$ and $U\left(L_{O}, y, t\right)=0$. The meridional axis $y$ and $Y$ are different in the atmosphere and ocean as they each scale to a suitable Rossby radius, which allows for a systematic meridional decomposition of the system into the well-known parabolic cylinder functions (Majda 2003). In practice, we retain and solve the components only for the first atmosphere and ocean parabolic cylinder functions, which keeps the system low dimensional (see supplementary information of Thual et al. 2016). The dimensional reference scales are $x: 15000 \mathrm{~km}, y: 1500 \mathrm{~km}, Y: 330 \mathrm{~km}, t: 3.3$ days, $u: 5 \mathrm{~m} \mathrm{~s}^{-1}$, and $\theta, q: 1.5 \mathrm{~K}$ (see Thual et al. 2016). Table B1 defines all parameters used in the model and provides their nondimensional values. In addition, the zonal profile of the thermocline feedback parameter $\eta(x)$ is shown in Fig. 2c. All parameter values are identical to the ones of Thual et al. (2016), except for a few additional parameters of the intraseasonal atmosphere: $s^{q}$ and $s^{\theta}$ (see Fig. 2), $\Gamma=1.66\left(\approx 0.3 \mathrm{~K}^{-1} \mathrm{day}^{-1}\right.$ as in Thual et al. 2014), and $d_{u}, d_{\theta}, d_{q}, d_{a}, \lambda=(30 \text { day })^{-1}$ as well as $\sigma_{q}=0.4$.

As regards the numerical solving algorithm, we use a simple split method to update the TSS-GCM. First, the interannual atmosphere and ocean are solved in a fashion identical to Thual et al. (2016) using the method of lines in space and Euler in time. Next, the intraseasonal atmosphere is solved in Fourier space in a fashion similar to Thual et al. (2014). The spatial resolution is $625 \mathrm{~km}$ and the temporal resolution is $0.8 \mathrm{~h}$. Numerical solutions span around 2000 years for each experiment presented in the present article, with a statistical equilibrium quickly reached after around 10 years starting from arbitrary initial conditions. It takes around $3 \mathrm{~h}$ to compute 2000 years of simulation on a personal laptop, which is computationally very uncostly.

\section{REFERENCES}

An, S.-I., and F.-F. Jin, 2001: Collective role of thermocline and zonal advective feedbacks in the ENSO mode. J. Climate, 14, 3421-3432, https://doi.org/10.1175/1520-0442(2001)014<3421: CROTAZ $>2.0 . \mathrm{CO} ; 2$.

Ashok, K., S. K. Behera, S. A. Rao, H. Weng, and T. Yamagata, 2007: El Niño Modoki and its possible teleconnections. J. Geophys. Res., 112, C11007, https://doi.org/10.1029/2006JC003798.

Chen, D., and Coauthors, 2015: Strong influence of westerly wind bursts on El Niño diversity. Nat. Geosci., 8, 339-345, https:// doi.org/10.1038/ngeo2399.

Chen, N., and A. J. Majda, 2016a: Filtering the stochastic skeleton model for the Madden-Julian oscillation. Mon. Wea. Rev., 144, 501-527, https://doi.org/10.1175/MWR-D-15-0261.1.

$\ldots$, and _ 2016b: Simple dynamical models capturing the key features of the central pacific El Niño. Proc. Natl. Acad. Sci. USA, 113, 11 732-11 737, https://doi.org/10.1073/pnas.1614533113.

— ing the statistical diversity of El Niño Southern Oscillation. Proc. Natl. Acad. Sci. USA, 114, 1468-1473, https://doi.org/ 10.1073/pnas.1620766114.

,$- \ldots$, and S. Thual, 2018: Observations and mechanisms of a simple stochastic dynamical model capturing El Niño diversity. J. Climate, 31, 449-471, https://doi.org/10.1175/JCLI-D-16-0880.1.

Chen, S., A. J. Majda, and S. N. Stechmann, 2016: Tropicalextratropical interactions with the MJO skeleton and climatological mean flow. J. Atmos. Sci., 73, 4101-4116, https:// doi.org/10.1175/JAS-D-16-0041.1.

Christensen, H. M., J. Berner, D. R. B. Coleman, and T. N. Palmer, 2017: Stochastic parameterization and El Niño-Southern Oscillation. J. Climate, 30, 17-38, https://doi.org/10.1175/ JCLI-D-16-0122.1. 
Clarke, A. J., 2008: An Introduction to the Dynamics of El Niño and the Southern Oscillation. Academic Press, $324 \mathrm{pp}$.

Deng, Q., B. Khouider, and A. J. Majda, 2015: The MJO in a coarse-resolution GCM with a stochastic multicloud parameterization. J. Atmos. Sci., 72, 55-74, https://doi.org/ 10.1175/JAS-D-14-0120.1.

Eisenman, I., L. Yu, and E. Tziperman, 2005: Westerly wind bursts: ENSO's tail rather than the dog? J. Climate, 18, 5224-5238, https://doi.org/10.1175/JCLI3588.1.

Fedorov, A., S. Hu, M. Lengaigne, and E. Guilyardi, 2015: The impact of westerly wind bursts and ocean initial state on the development, and diversity of El Niño events. Climate Dyn., 44, 1381-1401, https://doi.org/10.1007/s00382-014-2126-4.

Gebbie, G., I. Eisenman, A. Wittenberg, and E. Tziperman, 2007: Modulation of westerly wind bursts by sea surface temperature: A semistochastic feedback for ENSO. J. Atmos. Sci., 64, 3281-3295, https://doi.org/10.1175/JAS4029.1.

Gill, A., 1980: Some simple solutions for heat-induced tropical circulation. Quart. J. Roy. Meteor. Soc., 106, 447-462, https:// doi.org/10.1002/qj.49710644905.

Goswami, B. B., B. Khouider, R. Phani, P. Mukhopadhyay, and A. Majda, 2017a: Improving synoptic and intraseasonal variability in CFSv2 via stochastic representation of organized convection. Geophys. Res. Lett., 44,1104-1113, https://doi.org/ 10.1002/2016GL071542.

,,,$--- \ldots$, and $\_, 2017 \mathrm{~b}$ : Improved tropical modes of variability in the NCEP Climate Forecast System (version 2) via a stochastic multicloud model. J. Atmos. Sci., 74, 3339_ 3366, https://doi.org/10.1175/JAS-D-17-0113.1.

Guilyardi, E., A. Wittenberg, M. Balmaseda, W. Cai, M. Collins, M. J. McPhaden, M. Watanabe, and S.-W. Yeh, 2016: Fourth CLIVAR workshop on the evaluation of ENSO processes in climate models: ENSO in a changing climate. Bull. Amer. Meteor. Soc., 97, 817-820, https://doi.org/10.1175/ BAMS-D-15-00287.1.

Gushchina, D., and B. Dewitte, 2011: The relationship between intraseasonal tropical variability and ENSO and its modulation at seasonal to decadal timescales. Cent. Eur. J. Geosci., 3, 175-196, https://doi.org/10.2478/s13533-011-0017-3.

Harrison, D., and G. Vecchi, 1997: Westerly wind events in the tropical Pacific. J. Climate, 10, 3131-3156, https://doi.org/ 10.1175/1520-0442(1997)010<3131:WWEITT>2.0.CO;2.

Hendon, H. H., C. Zhang, and J. Glick, 1999: Interannual variation of the Madden-Julian oscillation during austral summer. J. Climate, 12, 2538-2550, https://doi.org/10.1175/ 1520-0442(1999)012<2538:IVOTMJ>2.0.CO;2.

- M. C. Wheeler, and C. Zhang, 2007: Seasonal dependence of the MJO-ENSO relationship. J. Climate, 20, 531-543, https:// doi.org/10.1175/JCLI4003.1.

Hottovy, S., and S. N. Stechmann, 2015: A spatiotemporal stochastic model for tropical precipitation and water vapor dynamics. J. Atmos. Sci., 72, 4721-4738, https://doi.org/10.1175/ JAS-D-15-0119.1.

Hu, S., and A. V. Fedorov, 2017: The extreme El Niño of 20152016: The role of westerly and easterly wind bursts, and preconditioning by the failed 2014 event. Climate Dyn., https:// doi.org/10.1007/s00382-017-3531-2.

$\ldots, \ldots$, M. Lengaigne, and E. Guilyardi, 2014: The impact of westerly wind bursts on the diversity and predictability of El Niño events: An ocean energetics perspective. Geophys. Res. Lett., 41, 4654-4663, https://doi.org/10.1002/2014GL059573.

Illig, S., and B. Dewitte, 2006: Local coupled equatorial variability versus remote ENSO forcing in an intermediate coupled model of the tropical Atlantic. J. Climate, 19, 5227-5252, https://doi.org/10.1175/JCLI3922.1.

Jin, F.-F., L. Lin, A. Timmermann, and J. Zhao, 2007: Ensemblemean dynamics of the ENSO recharge oscillator under statedependent stochastic forcing. Geophys. Res. Lett., 34, L03807, https://doi.org/10.1029/2006GL027372.

Kalnay, E., and Coauthors, 1996: The NCEP/NCAR 40-Year Reanalysis Project. Bull. Amer. Meteor. Soc., 77, 437-471, https:// doi.org/10.1175/1520-0477(1996)077<0437:TNYRP>2.0.CO;2.

Kiladis, G. N., C. Wheeler, P. T. Haertel, K. H. Straub, and P. E. Roundy, 2009: Convectively coupled equatorial waves. Rev. Geophys., 47, RG2003, https://doi.org/10.1029/2008RG000266.

Kim, D., and Coauthors, 2009: Application of MJO simulation diagnostics to climate models. J. Climate, 22, 6413-6436, https:// doi.org/10.1175/2009JCLI3063.1.

Kleeman, R., 2008: Stochastic theories for the irregularity of ENSO. Philos. Trans. Roy. Soc., 366A, 2509-2524, https://doi.org/ 10.1098/rsta.2008.0048.

— , and A. M. Moore, 1997: A theory for the limitation of ENSO predictability due to stochastic atmospheric transients. J. Atmos. Sci., 54, 753-767, https://doi.org/10.1175/ 1520-0469(1997)054<0753:ATFTLO>2.0.CO;2.

Lin, J.-L., and Coauthors, 2006: Tropical intraseasonal variability in 14 IPCC AR4 climate models. Part I: Convective signals. J. Climate, 19, 2665-2690, https://doi.org/10.1175/JCLI3735.1.

Lopez, H., B. Kirtman, E. Tziperman, and G. Gebbie, 2013: Impact of interactive westerly wind bursts on CCSM3. Dyn. Atmos. Oceans, 59, 24-51, https://doi.org/10.1016/j.dynatmoce.2012.11.001.

Madden, R. A., and P. R. Julian, 1971: Detection of a 40-50 day oscillation in the zonal wind in the tropical Pacific. J. Atmos. Sci., 28, 702-708, https://doi.org/10.1175/1520-0469(1971)028<0702: DOADOI $>2.0 . \mathrm{CO} ; 2$

- and - 1994: Observations of the 40-50 day tropical oscillation-A review. Mon. Wea. Rev., 122, 814-837, https:// doi.org/10.1175/1520-0493(1994)122<0814:OOTDTO >2.0.CO;2.

Majda, A., 2003: Introduction to PDEs and Waves for the Atmosphere and Ocean. Vol. 9. American Mathematical Society, 234 pp.

—, and R. Klein, 2003: Systematic multiscale models for the tropics. J. Atmos. Sci., 60, 393-408, https://doi.org/10.1175/ 1520-0469(2003)060<0393:SMMFTT $>2.0 . \mathrm{CO} ; 2$.

— , and S. N. Stechmann, 2009: The skeleton of tropical intraseasonal oscillations. Proc. Natl. Acad. Sci. USA, 106, 84178422, https://doi.org/10.1073/pnas.0903367106.

, and - , 2011: Nonlinear dynamics and regional variations in the MJO skeleton. J. Atmos. Sci., 68, 3053-3071, https:// doi.org/10.1175/JAS-D-11-053.1.

, C. Franzke, and B. Khouider, 2008: An applied mathematics perspective on stochastic modelling for climate. Philos. Trans. Roy. Soc., 366A, 2427-2453, https://oi.org/ 10.1098/rsta.2008.0012.

- S. Stechmann, R. Ogrosky, C. Chen, and S. Thual, 2018: Explaining tropical intraseasonal variability with the stochastic skeleton model. SpringerBriefs in Mathematics of Planet Earth: Weather, Climate, Oceans, C. J. Cotter et al., Eds., Springer, in press.

McPhaden, M. J., X. Zhang, H. H. Hendon, and M. C. Wheeler, 2006: Large scale dynamics and MJO forcing of ENSO variability. Geophys. Res. Lett., 33, L16702, https://doi.org/10.1029/ 2006GL026786.

Moore, A. M., and R. Kleeman, 1999: Stochastic forcing of ENSO by the intraseasonal oscillation. J. Climate, 12, 1199-1220, https:// doi.org/10.1175/1520-0442(1999)012<1199:SFOEBT>2.0.CO;2. 
Neelin, J. D., and N. Zeng, 2000: A quasi-equilibrium tropical circulation model-Formulation. J. Atmos. Sci., 57, 17411766, https://doi.org/10.1175/1520-0469(2000)057<1741: $\mathrm{AQETCM}>2.0 . \mathrm{CO} ; 2$.

— D. S. Battisti, A. C. Hirst, F.-F. Jin, Y. Wakata, T. Yamagata, and S. E. Zebiak, 1998: ENSO theory. J. Geophys. Res., 103, 14 261-14 290, https://doi.org/10.1029/97JC03424.

Ogrosky, H. R., and S. N. Stechmann, 2015: The MJO skeleton model with observation-based background state and forcing. Quart. J. Roy. Meteor. Soc., 141, 2654-2669, https://doi.org/ 10.1002/qj.2552.

Palmer, T. N., 2012: Towards the probabilistic Earth-system simulator: A vision for the future of climate and weather prediction. Quart. J. Roy. Meteor. Soc., 138, 841-861, https://doi.org/ 10.1002/qj.1923.

Puy, M., J. Vialard, M. Lengaigne, and E. Guilyardi, 2016: Modulation of equatorial Pacific westerly/easterly wind events by the Madden-Julian oscillation and convectively-coupled Rossby waves. Climate Dyn., 46, 2155-2178, https://doi.org/ 10.1007/s00382-015-2695-x.

Santoso, A., M. J. McPhaden, and W. Cai, 2017: The defining characteristics of ENSO extremes and the strong 2015/2016 El Niño. Rev. Geophys., 55, 1079-1129, https://doi.org/10.1002/ 2017RG000560.

Stachnik, J. P., D. E. Waliser, A. J. Majda, S. N. Stechmann, and S. Thual, 2015: Evaluating MJO event initiation and decay in the skeleton model using an RMM-like index. J. Geophys. Res. Atmos., 120, 11 486-11 508, https://doi.org/10.1002/2015JD023916.

Stechmann, S. N., and A. J. Majda, 2015: Identifying the skeleton of the Madden-Julian oscillation in observational data. Mon. Wea. Rev., 143, 395-416, https://doi.org/10.1175/ MWR-D-14-00169.1.

-, and S. Hottovy, 2017: Unified spectrum of tropical rainfall and waves in a simple stochastic model. Geophys. Res. Lett., 44, 10713-10 724, https://doi.org/10.1002/2017GL075754.

Thual, S., A. J. Majda, and S. N. Stechmann, 2014: A stochastic skeleton model for the MJO. J. Atmos. Sci., 71, 697-715, https://doi.org/10.1175/JAS-D-13-0186.1.

,-- , and - 2015: Asymmetric intraseasonal events in the skeleton MJO model with seasonal cycle. Climate Dyn., 45, 603-618, https://doi.org/10.1007/s00382-014-2256-8.

, ——, N. Chen, and S. N. Stechmann, 2016: Simple stochastic model for El Niño with westerly wind bursts. Proc. Natl. Acad. Sci. USA, 113, 10 245-10 250, https://doi.org/10.1073/pnas.1612002113.
,-- , and -2017 : Seasonal synchronization of a simple stochastic dynamical model capturing El Niño diversity. J. Climate, 30, $10047-10$ 066, https://doi.org/10.1175/ JCLI-D-17-0174.1.

- —_, and — 2018: Statistical occurrence and mechanisms of the 2014-2016 delayed super El Niño captured by a simple dynamical model. Climate Dyn., https://doi.org/10.1007/ s00382-018-4265-5, in press.

Tziperman, E., and L. Yu, 2007: Quantifying the dependence of westerly wind bursts on the large-scale tropical Pacific SST. J. Climate, 20, 2760-2768, https://doi.org/10.1175/JCLI4138a.1.

Vecchi, G., and D. Harrison, 2000: Tropical Pacific sea surface temperature anomalies, El Niño, and equatorial westerly wind events. J. Climate, 13, 1814-1830, https://doi.org/10.1175/ 1520-0442(2000)013<1814:TPSSTA >2.0.CO;2.

Weisheimer, A., S. Corti, T. Palmer, and F. Vitart, 2014: Addressing model error through atmospheric stochastic physical parametrizations: Impact on the coupled ECMWF seasonal forecasting system. Philos. Trans. Roy. Soc., 372A, 20130290, https://doi.org/10.1098/rsta.2013.0290.

Wheeler, M., and G. N. Kiladis, 1999: Convectively coupled equatorial waves: Analysis of clouds and temperature in the wavenumberfrequency domain. J. Atmos. Sci., 56, 374-399, https://doi.org/ 10.1175/1520-0469(1999)056<0374:CCEWAO > 2.0.CO;2.

Wittenberg, A. T., A. Rosati, N.-C. Lau, and J. Ploshay, 2006: GFDL's CM2 global coupled climate models. Part III: Tropical Pacific climate and ENSO. J. Climate, 19, 698-722, https:// doi.org/10.1175/JCLI3631.1.

,-- T. L. Delworth, G. A. Vecchi, and F. Zeng, 2014: ENSO modulation: Is it decadally predictable? J. Climate, 27, 26672681, https://doi.org/10.1175/JCLI-D-13-00577.1.

Zebiak, S., and M. A. Cane, 1987: A model El Niño-Southern Oscillation. Mon. Wea. Rev., 115, 2262-2278, https://doi.org/ 10.1175/1520-0493(1987)115<2262:AMENO>2.0.CO;2.

Zeng, N., J. D. Neelin, and C. Chou, 2000: A quasi-equilibrium tropical circulation model-implementation and simulation. J. Atmos. Sci., 57, 1767-1796, https://doi.org/10.1175/ 1520-0469(2000)057<1767:AQETCM>2.0.CO;2.

Zhang, C., 2005: Madden-Julian oscillation. Rev. Geophys., 43, RG2003, https://doi.org/10.1029/2004RG000158. and J. Gottschalck, 2002: SST anomalies of ENSO and the Madden-Julian oscillation in the equatorial Pacific. J. Climate, 15, 2429-2445, https://doi.org/10.1175/1520-0442(2002)015<2429: SAOEAT $>2.0 . \mathrm{CO} ; 2$. 